\title{
ORDENAÇÃO DE POPULAÇÕES EM AMPLAS CLASSES DE NIVEL DE SAUDE, SEGUNDO UM INDICADOR ABRANGENTE DEFINIDO POR UMA FUNÇÃO DISCRIMINANTE LINEAR
}

\author{
Neil Ferreira Novo* \\ Yára Juliano* \\ Elias Rodrigues de Paiva* \\ Walter Leser*
}

Novo, N.F. et al. Ordenação de populaçōes em amplas classes de nível de saúde, se-
gundo um indicador abrangente definido por uma função discriminante linear. Rev.
Saúde públ., S. Paulo, $19: 344-63,1985$.

RESUMO: Utilizando a função discriminante linear, propõe-se um indicador de nível de saúde abrangente de vários indicadores usuais, a saber: o coeficiente de mortalidade geral (CMG), indicador quantificado de Guedes (IG), esperança de vida ao nascer $(\mathrm{EV})$, coeficiente de natalidade $(\mathrm{CN})$, coeficiente de mortalidade infantil (CMI) e coeficiente de mortalidade por doenças transmissíveis (CMDT). Para a padronização dos dois últimos, foi proposta e utilizada uma população padrão mediana; para sua formação, cada grupo etário concorre com a mediana das percentagens de participação desse grupo na composição da população de cada um dos 44 países estudados. A análise crítica das equações de funções discriminantes obtidas com a técnica passo a passo ascendente (stepwise), mostrou que o valor: $Z=\frac{2895}{\mathrm{CMI}}+\frac{2060}{\mathrm{CN}}+\frac{1000}{\mathrm{CMDTp}}$, pode ser utilizado como indicador abrangente, permitindo a ordenação de países em amplas classes de nível de saúde.

UNITERMOS: Indicador de nível de saúde. Função discriminante linear. População padrão mediana.

\section{INTRODUÇÃO}

Para avaliação do nível de saúde de uma população são utilizados vários indicadores, na sua quase totalidade representados por coeficientes baseados em dados de mortalidade (geral, por doenças transmissiveis, infantil e materna) ou em valores da mortalidade proporcional por idade (Razão de Mortalidade Proporcional de Swaroop e Uemura, Curva de Mortalidade Proporcional de Nelson Moraes e Indicador Quantificado de Guedes). Também calculadas a partir de dados de mortalidade, frequientemente são referidas as esperanças de vida, ao nas- cer e na idade de um ano. Uma exceção é dada pelo coeficiente de natalidade, demonstradamente relacionada com as condições de saúde do grupo maternoinfantil.

Verifica-se porém, com frequiência que, quando são comparadas várias populações utilizando cada um desses indicadores, observam-se discrepâncias, por vezes marcantes, entre as posições relativas que um mesmo país ocupa nas diferentes ordenações. Assim, de uma comparação realizada em termos de um de-

- Do Departamento de Medicina Preventiva da Escola Paulista de Medicina - Rua Botucatu. 740 -. 04023 - Sāo Paulo, SP - Brasil. 
NOVO, N.F. et al. Ordenação de populações em amplas classes de nível de saúde, segundo um indicador abrangente definido por uma função discriminante linear. Rev. Saúde públ. S. Paulo, 19:344-63, 1985.

les, resultarão conclusões que, na realidade, dependem da escolha feita.

Recorrendo ao emprego de técnica já utilizada por Swaroop e Uemura ${ }^{10}(1957)$ procurou-se estudar as equações discriminantes envolvendo vários indicadores mais frequientemente utilizados, visando a encontrar, entre elas, a que mais adequadamente forneça uma expressão abrangente do conjunto de indicadores estudados. Com os valores da estatística $Z$, calculados, segundo tal equação, torna-se possível a ordenação em amplas classes, quanto ao nível de saúde, de diferentes populações, ou de uma mesma população em sucessivas épocas.

\section{MATERIAL E METODOS}

\section{Indicadores utilizados}

$\begin{array}{cl}\text { I - Indicadores } & \text { Coeficiente de } \\ \text { mortobais } & \text { (CMG) } \\ & \text { Indicador de } \\ & \text { Guedes (IG) } \\ & \text { Esperança de vida } \\ & \text { ao nascer (EV) } \\ & \text { Coeficiente de } \\ & \text { natalidade (CN) }\end{array}$

II - Indicadores Coeficiente de Específicos mortalidade infantil (CMI)

Coeficiente de mortalidade por doenças transmissiveis (CMDT)

Os valores de $\mathrm{CN}$ e do $\mathrm{CMI}$ foram colhidos diretamente das fontes consultadas. Os valores da EV foram fornecidos nas fontes, separadamente para homens e para mulheres; o valor para a população foi obtido pelo cálculo da média ponderada dos valores para os sexos.
Para o cálculo do IG surgiu o problema da discrepância entre a distribuição etária dos óbitos, adotada no anuário da OMS ${ }^{11}$, e os grupos etários propostos por Moraes ${ }^{6}$ (1959) e seguidos por Guedes ${ }^{4}$ (1972). Tornaram-se necessárias interpolações, nos grupos 15-24 e 45-54, tendo-se para tanto recorrido aos multiplicadores de Karup-King indicados para a divisão de grupos ao meio (Shryock e Siegel 7, 1973).

No caso do CMG impunha-se a padronização, tendo em vista as previsíveis diferenças de composição das populações. A mesma situação ocorria, evidentemente, com relação ao CMDT, acrescentando-se, entretanto, a necessidade de ser considerada a principal das causas de erro envolvidas na determinação deste coeficiente, ou seja, a frequiência de óbitos classificados na rubrica "sintomas, sinais e afecções mal definidas". Referirnos-emos a eles, como é usual, por "óbitos por causas mal definidas".

\section{PAISES ESTUDADOS}

Para a inclusão de um país no conjunto a ser estudado, era necessário que se dispusesse de dados referentes à população e aos óbitos por causas, com distribuição por grupos etários, bem como de informações sobre o valor da natalidade e da esperança de vida ao nascer, em um dado ano.

Como fonte básica de tais dados foram utilizadas edições sucessivas de publicação da OMS (World Health Statistics Annual ${ }^{11}$, 1977-1982).

Estabeleceu-se, ainda, que somente seriam incluídos países cujos dados fossem apontados, na publicação referida, como confiáveis. 
NOVO, N.F. et al. Ordenação de populações em amplas classes de nível de saúde, segundo um indicador abrangente definido por uma função discriminante linear. Rev. Saúde públ., S. Paulo, 19:344-63, 1985.

Em alguns poucos casos, dados faltantes puderam ser encontrados em edições sucessivas de publicação das Nações Unidas (Demographic Yearbook, 19781981).

Procurou-se utilizar dados recentes, remontando o mais antigo (México) a 1975.

Levando em conta os critérios acima descritos, chegou-se a uma amostra de 44 países, estes com os mais variados graus de desenvolvimento e de tamanho de população, com localização geográfica abrangendo cinco continentes. Não se trata, evidentemente, de amostra representativa da totalidade dos países, pois a inclusão de cada um dependeu da sua capacidade de fornecer, à OMS, dados por esta qualificados como confiáveis. Tal característica, a da representatividade, não era, entretanto, necessária para o objetivo do presente estudo. Infelizmente, não pôde ser incluído o Brasil, por não figurarem seus dados nas fontes utilizadas.

Foram incluídos no estudo os países relacionados em ordem alfabética na $\mathrm{Ta}$ bela 1 , na qual constam, também, para cada um dos grupos etários adotados nas publicações da OMS, os dados referentes a população e a participação percentual no total. Sendo os dados apresentados nas fontes com arredondamento ao milhar, as somas dos valores dos grupos etários podem não coincidir exatamente com a população total. $\mathrm{O}$ mesmo sucede, naturalmente, no caso das percentagens de participação, calculadas com arredondamento à segunda decimal. Apenas no caso do Japão foi encontrada, nas fontes, frequiência na casela referente a "idade não especificada"; procedeu-se, então, à distribuição dessa frequiência (54.000) em partes proporcionais às freqüências dos grupos etários.
Correção da causa de erro decorrente de óbitos por causas mal definidas

Tentando atenuar os erros que resultam, no cálculo do CMDT, de diferenças marcantes, entre os países, nas frequiências relativas de óbitos por causas mal definidas, foi necessário trabalhar com a hipótese de que, entre eles, a proporção de óbitos por doenças transmissíveis seja a mesma que a existente entre os que têm causas identificadas nos atestados, ou seja, o total de óbitos menos os óbitos por causas mal definidas.

Com essa admissão e sendo:

0 = total de óbitos;

$\mathrm{t}=$ número de óbitos por doenças transmissíveis (segundo os atestados);

$\mathrm{MD}=$ número de óbitos por causas mal definidas,

temos:

$\frac{t}{0-\mathrm{MD}}=\begin{aligned} & \text { proporção de óbitos por } \\ & \text { doenças transmissíveis entre } \\ & \begin{array}{l}\text { os que têm causas identifica- } \\ \text { das; }\end{array}\end{aligned}$

txMD número de óbitos por doen$\longrightarrow=$ ças transmissíveis entre os 0-MD óbitos por causas mal definidas;

$T=t+\frac{{ }^{x M D}}{0-M D}=\frac{t_{0}}{0-M D}=$

total estimado, em função da admissão feita, de óbitos por doenças transmissíveis.

Esse total, calculado por grupo etário, foi utilizado na padronização do coeficiente. 
NOVO, N.F. et al. Ordenação de populações em amplas classes dis nível de saúde, segundo um indicador abrangente definido por uma função discriminante linear. Rev. Saúde públ., S. Paulo, 19:344-63, 1985.

TABELA 1

População (x 1000) e percentagem de participação segundo grupos etários e países, nos anos especificados

\begin{tabular}{|c|c|c|c|c|c|c|c|c|c|c|c|c|c|}
\hline \multirow{2}{*}{ PAIS } & \multirow{2}{*}{ ANO } & \multirow{2}{*}{$\begin{array}{l}\text { MaTUREZA } \\
\text { Dos DADOS* }\end{array}$} & \multicolumn{10}{|c|}{ GRUPOS ETRRIOS } & \multirow{2}{*}{ TOTAL } \\
\hline & & & 1 & 1.4 & -14 & 15.24 & $25-34$ & \begin{tabular}{|l|}
55.44 \\
\end{tabular} & 45.54 & 55.64 & 165.74 & $75 \varepsilon+$ & \\
\hline Alenanha & 1980 & p & $\begin{array}{l}598 \\
0.97\end{array}$ & $\begin{array}{r}2332 \\
3,79\end{array}$ & $\begin{array}{l}8253 \\
13,41\end{array}$ & $\begin{array}{l}9877 \\
16,05\end{array}$ & $\begin{array}{l}8268 \\
13,43\end{array}$ & $\begin{array}{l}9177 \\
14,91\end{array}$ & $\begin{array}{l}7569 \\
12,33\end{array}$ & $\begin{array}{r}5907 \\
9,60\end{array}$ & $\begin{array}{l}5966 \\
9,69\end{array}$ & $\begin{array}{l}3583 \\
5,82\end{array}$ & $\begin{array}{l}61549 \\
100,00\end{array}$ \\
\hline Austräl is & 1980 & $\stackrel{p}{q}$ & $\underset{1,52}{222}$ & $\begin{array}{l}909 \\
6,22\end{array}$ & $\begin{array}{l}2567 \\
17,56\end{array}$ & $\begin{array}{l}2549 \\
17,44\end{array}$ & $\begin{array}{l}2360 \\
16,15\end{array}$ & $\begin{array}{l}1768 \\
12,10\end{array}$ & $\begin{array}{l}\begin{array}{r}518 \\
10,39\end{array} \\
\end{array}$ & $\begin{array}{l}1321 \\
9,04\end{array}$ & $\begin{array}{l}904 \\
6,19\end{array}$ & $\begin{array}{l}498 \\
3.44\end{array}$ & $\begin{array}{l}\begin{array}{l}14616 \\
100,00\end{array} \\
0\end{array}$ \\
\hline Austria & 1980 & $\stackrel{p}{q}$ & $\begin{array}{r}89 \\
1,19\end{array}$ & $\begin{array}{r}345 \\
4,60\end{array}$ & $\begin{array}{l}1,107 \\
14,75\end{array}$ & $\begin{array}{l}1225 \\
16,32\end{array}$ & ${ }_{13,56}^{018}$ & $\begin{array}{r}983 \\
13,10\end{array}$ & $\begin{array}{r}846 \\
11,27\end{array}$ & $\begin{array}{l}732 \\
9,75\end{array}$ & $\begin{array}{l}711 \\
8,47\end{array}$ & $\begin{array}{l}450 \\
6,00\end{array}$ & $\begin{array}{r}7505 \\
100,00\end{array}$ \\
\hline Barbados & 1979 & $\stackrel{p}{:}$ & 1,59 & 6.17 & $\begin{array}{l}502 \\
20,72\end{array}$ & $\begin{array}{l}58 \\
23,11\end{array}$ & $\begin{array}{r}38 \\
15,14\end{array}$ & 7,59 & 7,59 & $, 1,57$ & $\begin{array}{l}15 \\
6,98\end{array}$ & $\begin{array}{r}3,1^{8} \\
3,19\end{array}$ & $\begin{array}{r}251 \\
100,00\end{array}$ \\
\hline Bëlgíca & 1977 & $\stackrel{p}{x}$ & $\begin{array}{l}120 \\
1,22\end{array}$ & $\begin{array}{r}497 \\
5,06\end{array}$ & $\begin{array}{l}1484 \\
15,10\end{array}$ & $\begin{array}{l}\mathfrak{1}_{15,862}^{562} \\
\end{array}$ & $\begin{array}{l}1396 \\
14,20\end{array}$ & $\begin{array}{l}166 \\
11,86\end{array}$ & $\left\{\begin{array}{r}2,1 \\
12,93\end{array}\right.$ & $\begin{array}{l}953 \\
9,69\end{array}$ & $\begin{array}{l}862 \\
8.77\end{array}$ & $\begin{array}{r}519 \\
5,28\end{array}$ & $\begin{array}{r}9830 \\
100,00\end{array}$ \\
\hline Bulgäria & 1980 & $\stackrel{p}{?}$ & $\begin{array}{l}129 \\
1,46\end{array}$ & $\begin{array}{r}548 \\
6,18\end{array}$ & $\begin{array}{l}1285 \\
14,50\end{array}$ & $\begin{array}{l}1253 \\
14,14\end{array}$ & $\begin{array}{l}1321 \\
14,91\end{array}$ & $\begin{array}{l}120 \\
12,64\end{array}$ & 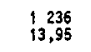 & $\begin{array}{l}918 \\
10,36\end{array}$ & $\begin{array}{l}7,2 \\
8,03\end{array}$ & $\begin{array}{r}340 \\
3.84\end{array}$ & $\begin{array}{l}8862 \\
100,00\end{array}$ \\
\hline Canadis & 1978 & $\stackrel{p}{:}$ & $\begin{array}{l}356 \\
1,52\end{array}$ & $\begin{array}{l}1,392 \\
5,93\end{array}$ & $\begin{array}{l}3937 \\
16,76\end{array}$ & $\begin{array}{l}{ }_{19}^{4634} \\
19.73\end{array}$ & $\begin{array}{l}3839 \\
18,34\end{array}$ & $\begin{array}{l}2691 \\
11,46\end{array}$ & $\begin{array}{l}2.499 \\
10,55\end{array}$ & $\begin{array}{l}2024 \\
8,62\end{array}$ & $\begin{array}{l}1340 \\
5,70\end{array}$ & $\begin{array}{r}795 \\
3,38\end{array}$ & $\begin{array}{l}23490 \\
100,00\end{array}$ \\
\hline ile & 1979 & $\stackrel{p}{q}$ & $\begin{array}{l}2.66 \\
2,44\end{array}$ & $\begin{array}{l}980 \\
8,98\end{array}$ & $\begin{array}{l}2.355 \\
21,57\end{array}$ & $\begin{array}{l}2281 \\
20,89\end{array}$ & $\begin{array}{l}{ }_{1}^{665} \\
15,25\end{array}$ & $\begin{array}{l}1232 \\
11,28\end{array}$ & $\begin{array}{l}919 \\
8,42\end{array}$ & $\begin{array}{l}629 \\
5,76\end{array}$ & $\begin{array}{r}398 \\
3,65\end{array}$ & $\begin{array}{l}193 \\
1,77\end{array}$ & $\begin{array}{l}10918 \\
100,00\end{array}$ \\
\hline ta $R_{i}$ & 1979 & $\stackrel{p}{a}$ & $\begin{array}{l}70 \\
3,23\end{array}$ & $\begin{array}{l}213 \\
9,82\end{array}$ & $\begin{array}{r}545 \\
25,12\end{array}$ & $\begin{array}{r}504 \\
23,23\end{array}$ & $\begin{array}{r}320 \\
14,75\end{array}$ & $\begin{array}{l}202 \\
9,31\end{array}$ & $\begin{array}{l}145 \\
6,68\end{array}$ & $\begin{array}{l}94 \\
4,33\end{array}$ & $\begin{array}{r}53 \\
2,44\end{array}$ & $\begin{array}{l}25 \\
1,15\end{array}$ & $\begin{array}{r}20170 \\
100,00\end{array}$ \\
\hline Dinama rea & 1980 & $\stackrel{p}{a}$ & $\begin{array}{r}58 \\
1,13\end{array}$ & $\begin{array}{r}256 \\
5,00\end{array}$ & $\begin{array}{r}754 \\
14,22\end{array}$ & $\begin{array}{r}267 \\
14,97\end{array}$ & $\begin{array}{r}787 \\
15,36\end{array}$ & $\begin{array}{r}671 \\
13,10\end{array}$ & $\begin{array}{l}546 \\
10,66\end{array}$ & $\begin{array}{r}546 \\
10,66\end{array}$ & $\begin{array}{l}448 \\
8.74\end{array}$ & $\begin{array}{l}290 \\
5,66\end{array}$ & $\begin{array}{c}5 \\
5123 \\
100,00\end{array}$ \\
\hline Egito & 1978 & $\stackrel{p}{q}$ & $\begin{array}{l}12,4 \\
3,05\end{array}$ & $\begin{array}{l}4263 \\
10,72\end{array}$ & 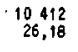 & $\begin{array}{l}76,64 \\
19,32\end{array}$ & $\begin{array}{l}5226 \\
13,14\end{array}$ & $\begin{array}{l}4270 \\
10,74\end{array}$ & $\begin{array}{l}3245 \\
8,16\end{array}$ & $\begin{array}{r}2023 \\
5,09\end{array}$ & $\begin{array}{l}1 \\
2,59\end{array}$ & $\begin{array}{l}400 \\
1.01\end{array}$ & $\begin{array}{l}39967 \\
100,00\end{array}$ \\
\hline Escōocia & 1981 & p & $\begin{array}{l}67 \\
,, 30\end{array}$ & $\begin{array}{r}248 \\
4,82\end{array}$ & $\begin{array}{r}786 \\
15,26\end{array}$ & $\begin{array}{l}86 t \\
76,72\end{array}$ & $\begin{array}{r}717 \\
13,92\end{array}$ & $\begin{array}{r}602 \\
11,69\end{array}$ & $\begin{array}{r}588 \\
11,42\end{array}$ & $\begin{array}{r}553 \\
10,74\end{array}$ & $\begin{array}{l}454 \\
8.82\end{array}$ & $\begin{array}{l}273 \\
5,30\end{array}$ & $\begin{array}{r}5150 \\
700,00\end{array}$ \\
\hline Espanha & 1978 & $\stackrel{p}{q}$ & $\begin{array}{l}643 \\
1,75\end{array}$ & $\begin{array}{r}2.652 \\
7,21\end{array}$ & $\begin{array}{l}5484 \\
17,63\end{array}$ & $\begin{array}{l}5.939 \\
18,15\end{array}$ & $\begin{array}{l}4920 \\
13,38\end{array}$ & ${ }_{11,71}^{405}$ & $\begin{array}{l}4520 \\
12,29\end{array}$ & $\begin{array}{l}3390 \\
9,22\end{array}$ & $\begin{array}{l}2,542 \\
8,91\end{array}$ & $\begin{array}{r}1,384 \\
3.76\end{array}$ & $\begin{array}{l}36778 \\
100,00\end{array}$ \\
\hline Estados unidos & 1977 & p & $\begin{array}{l}3,163 \\
1,46\end{array}$ & $\begin{array}{r}12078 \\
5,58\end{array}$ & $\begin{array}{r}36,372 \\
16,81\end{array}$ & $\begin{array}{r}40,993 \\
18,94\end{array}$ & $\begin{array}{l}33,004 \\
15,25\end{array}$ & $\begin{array}{r}23,48 t \\
10,85\end{array}$ & $\begin{array}{l}23,384 \\
10,81\end{array}$ & $\begin{array}{r}20 \\
9,402\end{array}$ & $\begin{array}{l}14.590 \\
6.74\end{array}$ & $\begin{array}{r}8,917 \\
4,12\end{array}$ & $\begin{array}{r}216383 \\
100,00\end{array}$ \\
\hline Fin| Êndia & 1978 & $\stackrel{p}{q}$ & $\begin{array}{l}64 \\
1,35\end{array}$ & $\begin{array}{r}252 \\
5,30\end{array}$ & $\begin{array}{r}682 \\
14,35\end{array}$ & $\begin{array}{r}784 \\
16,49\end{array}$ & $\begin{array}{r}830 \\
17,46\end{array}$ & $\begin{array}{r}578 \\
12,16\end{array}$ & $\begin{array}{r}557 \\
11,72\end{array}$ & $\begin{array}{l}458 \\
9,64\end{array}$ & $\begin{array}{l}372 \\
7,83\end{array}$ & $\begin{array}{l}176 \\
3,70\end{array}$ & $\begin{array}{r}4753 \\
100,00\end{array}$ \\
\hline Franca & 1978 & $\stackrel{p}{q}$ & $\begin{array}{l}734 \\
1,38\end{array}$ & $\begin{array}{r}3008 \\
5,65\end{array}$ & $\begin{array}{l}8026 \\
15,06\end{array}$ & $\begin{array}{l}8483 \\
15,92\end{array}$ & $\begin{array}{l}82,09 \\
15,41\end{array}$ & $\begin{array}{l}6037 \\
11,33\end{array}$ & $\begin{array}{l}{ }_{1}^{5} 475 \\
12,15\end{array}$ & $\begin{array}{r}4657 \\
8,74\end{array}$ & $\begin{array}{r}4.505 \\
8,46\end{array}$ & $\begin{array}{l}3,144 \\
5,90\end{array}$ & $\begin{array}{l}532,27 \\
100,00\end{array}$ \\
\hline Grêcia & 1979 & $?$ & $\begin{array}{l}145 \\
1,53\end{array}$ & $\begin{array}{r}563 \\
5,96\end{array}$ & $\begin{array}{l}1483 \\
15,69\end{array}$ & $\begin{array}{l}1397 \\
14,78\end{array}$ & 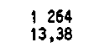 & $\begin{array}{l}1204 \\
12,74\end{array}$ & $\prod_{13,55}^{280}$ & $\begin{array}{l}881 \\
9,32\end{array}$ & $\begin{array}{l}777 \\
8,22\end{array}$ & $\begin{array}{l}456 \\
4,83\end{array}$ & $\begin{array}{l}9449 \\
100,00\end{array}$ \\
\hline Guat tenal & 198 & $\stackrel{p}{s}$ & $\begin{array}{l}267 \\
3,68\end{array}$ & $\begin{array}{l}1060 \\
14,60\end{array}$ & $\begin{array}{l}2258 \\
31,10\end{array}$ & $\begin{array}{l}1090 \\
15,01\end{array}$ & $\begin{array}{r}947 \\
13,04\end{array}$ & $\begin{array}{l}677 \\
9,33\end{array}$ & $\begin{array}{r}394 \\
5,43\end{array}$ & $\begin{array}{r}290 \\
3,99\end{array}$ & $\begin{array}{r}208 \\
2,87\end{array}$ & $\begin{array}{r}69 \\
0,95\end{array}$ & $\begin{array}{r}7260 \\
100,00\end{array}$ \\
\hline Guian & 1977 & $\stackrel{p}{:}$ & $\begin{array}{l}246 \\
2,96\end{array}$ & 11,75 & $\begin{array}{l}2936 \\
29,14\end{array}$ & 22,10 & $\begin{array}{r}93 \\
11,48\end{array}$ & $\begin{array}{r}67 \\
8,27\end{array}$ & $\begin{array}{r}52 \\
6,42\end{array}$ & $\begin{array}{l}\text { 37 } \\
4,5 ?\end{array}$ & $\begin{array}{r}20 \\
2,47\end{array}$ & 0,86 & $\begin{array}{r}810 \\
100,00\end{array}$ \\
\hline Holanda & 1980 & $\stackrel{p}{n}$ & $\begin{array}{l}1,18 \\
1,26\end{array}$ & $\begin{array}{l}707 \\
5,00\end{array}$ & $\begin{array}{l}2274 \\
16,07\end{array}$ & $\begin{array}{l}2456 \\
17,36\end{array}$ & $\begin{array}{l}2356 \\
16,66\end{array}$ & $\begin{array}{l}1740 \\
12,30\end{array}$ & $\begin{array}{l}1498 \\
10,59\end{array}$ & $\begin{array}{l}1311 \\
9,27\end{array}$ & $\begin{array}{l}987 \\
6,98\end{array}$ & $\begin{array}{l}642 \\
4,54\end{array}$ & $\begin{array}{l}14150 \\
100,00\end{array}$ \\
\hline Hong Kong & 1978 & $\stackrel{p}{q}$ & $\begin{array}{l}78 \\
1,69\end{array}$ & $\begin{array}{l}318 \\
6,90\end{array}$ & $\begin{array}{r}887 \\
19,26\end{array}$ & $\begin{array}{l}2056 \\
22,93\end{array}$ & $\begin{array}{r}690 \\
14.76\end{array}$ & $\begin{array}{r}458 \\
.9,94\end{array}$ & $\begin{array}{l}40,74 \\
10,73\end{array}$ & $\begin{array}{l}362 \\
7,86\end{array}$ & $\begin{array}{r}189 \\
4,10\end{array}$ & $\begin{array}{r}84 \\
1,82\end{array}$ & $\begin{array}{l}4506 \\
100,00\end{array}$ \\
\hline Hungria & 1980 & p & $\begin{array}{l}159 \\
1,43\end{array}$ & $\begin{array}{l}692 \\
6,46\end{array}$ & $\begin{array}{l}1504 \\
14,04\end{array}$ & ${ }_{13,37}^{433}$ & $\begin{array}{l}1575 \\
15,64\end{array}$ & $\begin{array}{l}\sqrt[372]{1372} \\
12,81\end{array}$ & $\left\{\begin{array}{l}368 \\
12,77\end{array}\right.$ & $\begin{array}{l}1076 \\
10,05\end{array}$ & $\begin{array}{l}951 \\
8,88\end{array}$ & $\begin{array}{l}4,58 \\
4,56\end{array}$ & $\begin{array}{l}10711 \\
100,00\end{array}$ \\
\hline
\end{tabular}


NOVO, N.F. et al. Ordenação de populações em amplas classes de nível de saúde, segundo um indicador abrangente definido por uma função discriminante linear. Rev. Saúde públ., S. Paulo, 19:344-63, 1985.

TABELA 1 - continuação

\begin{tabular}{|c|c|c|c|c|c|c|c|c|c|c|c|c|c|}
\hline \multirow{2}{*}{ PAIS } & \multirow{2}{*}{ ANO } & \multirow{2}{*}{$\begin{array}{l}\text { NATUREZA } \\
\text { DOS DADOS* }\end{array}$} & \multicolumn{10}{|c|}{ GRUPOS ETARIOS } & \multirow{2}{*}{ TOTAL } \\
\hline & & & $<1$ & $1-4$ & $5 \cdot 14$ & $15=24$ & $25-34$ & $35-44$ & $45-54$ & $55-64$ & $65-74$ & $75 \mathrm{E} *$ & \\
\hline t7has Mauricias & 1980 & P & $\begin{array}{r}25 \\
2,70\end{array}$ & $\begin{array}{r}88 \\
9,49\end{array}$ & 21,79 & $\begin{array}{r}214 \\
23,09\end{array}$ & $\begin{array}{r}149 \\
16,07\end{array}$ & $\begin{array}{r}86 \\
9,28\end{array}$ & $\begin{array}{r}71 \\
7,66\end{array}$ & $\begin{array}{r}53 \\
5,72\end{array}$ & $\begin{array}{r}28 \\
3,02\end{array}$ & $\begin{array}{r}11 \\
1,19\end{array}$ & $\begin{array}{r}927 \\
100,00\end{array}$ \\
\hline Inglaterra e Gales & 1980 & $\begin{array}{l}p \\
:\end{array}$ & $\begin{array}{r}644 \\
1,31\end{array}$ & $\begin{array}{r}2322 \\
4,72\end{array}$ & $\begin{array}{l}7325 \\
14,87\end{array}$ & $\begin{array}{l}7626 \\
15.49\end{array}$ & $\begin{array}{l}6999 \\
14,21\end{array}$ & $\begin{array}{l}5860 \\
11,90\end{array}$ & $\begin{array}{l}5554 \\
11,28\end{array}$ & $\begin{array}{l}5491 \\
11,15\end{array}$ & $\begin{array}{r}4611 \\
9,36\end{array}$ & $\begin{array}{r}2815 \\
5,72\end{array}$ & $\begin{array}{l}49246 \\
100,00\end{array}$ \\
\hline Irlanda & 1978 & $\begin{array}{l}P \\
:\end{array}$ & $\begin{array}{r}69 \\
2,98\end{array}$ & $\begin{array}{r}271 \\
8.18\end{array}$ & $\begin{array}{r}676 \\
20,40\end{array}$ & $\begin{array}{r}570 \\
17.20\end{array}$ & $\begin{array}{r}441 \\
13.31\end{array}$ & $\begin{array}{r}333 \\
10,05\end{array}$ & $\begin{array}{r}306 \\
9,23\end{array}$ & $\begin{array}{r}291 \\
8,78\end{array}$ & $\begin{array}{r}229 \\
6,91\end{array}$ & $\begin{array}{r}128 \\
3,86\end{array}$ & $\begin{array}{r}3314 \\
100,00\end{array}$ \\
\hline Irlanda do Norte & 1978 & p & $\begin{array}{r}25 \\
1,62\end{array}$ & $\begin{array}{r}101 \\
6,56\end{array}$ & $\begin{array}{r}299 \\
19,43\end{array}$ & $\begin{array}{r}267 \\
17,35\end{array}$ & $\begin{array}{r}200 \\
13,00\end{array}$ & $\begin{array}{r}168 \\
10,92\end{array}$ & $\begin{array}{r}158 \\
10,27\end{array}$ & $\begin{array}{r}145 \\
9,42\end{array}$ & $\begin{array}{r}1+5 \\
7,47\end{array}$ & $\begin{array}{r}61 \\
3,96\end{array}$ & $\begin{array}{r}1539 \\
100,00\end{array}$ \\
\hline Isländia & 1980 & $\stackrel{p}{:}$ & $\begin{array}{r}5 \\
2,19\end{array}$ & $\begin{array}{r}16 \\
7,02\end{array}$ & $\begin{array}{r}42 \\
18,42\end{array}$ & 19,34 & $\begin{array}{r}35 \\
15,35\end{array}$ & 10,53 & 9.65 & $\begin{array}{r}18 \\
7,89\end{array}$ & $\begin{array}{r}13 \\
5,70\end{array}$ & $\begin{array}{r}90 \\
4,39\end{array}$ & $\begin{array}{r}228 \\
100,00\end{array}$ \\
\hline [srael & 1980 & $\begin{array}{l}p \\
q\end{array}$ & $\begin{array}{r}93 \\
2,40\end{array}$ & $\begin{array}{r}381 \\
9.82\end{array}$ & $\begin{array}{r}815 \\
21,01\end{array}$ & $\begin{array}{r}670 \\
17,27\end{array}$ & $\begin{array}{r}621 \\
16,01\end{array}$ & $\begin{array}{r}360 \\
9,28\end{array}$ & $\begin{array}{r}327 \\
8,43\end{array}$ & $\begin{array}{r}278 \\
7,17\end{array}$ & $\begin{array}{r}229 \\
5,90\end{array}$ & $\begin{array}{r}106 \\
2,73\end{array}$ & $\begin{array}{r}3879 \\
100,00\end{array}$ \\
\hline Itälia & 1978 & $\begin{array}{l}P \\
\&\end{array}$ & $\begin{array}{r}729 \\
1,29\end{array}$ & $\begin{array}{r}3295 \\
5,89\end{array}$ & $\begin{array}{l}9058 \\
15,99\end{array}$ & $\begin{array}{l}8361 \\
14,74\end{array}$ & $\begin{array}{l}7808 \\
13,77\end{array}$ & $\begin{array}{l}7455 \\
13,14\end{array}$ & $\begin{array}{l}7220 \\
12,73\end{array}$ & $\begin{array}{r}5429 \\
9,57\end{array}$ & $\begin{array}{r}4,784 \\
8,44\end{array}$ & $\begin{array}{r}2565 \\
4,52\end{array}$ & $\begin{array}{l}56714 \\
100,00\end{array}$ \\
\hline tugoslävia & 1978 & $\stackrel{p}{*}$ & $\begin{array}{r}373 \\
1,70\end{array}$ & $\begin{array}{r}1,480 \\
6,74\end{array}$ & $\begin{array}{l}3618 \\
16,46\end{array}$ & $\begin{array}{l}3821 \\
17,39\end{array}$ & $\begin{array}{l}3280 \\
14,93\end{array}$ & $\begin{array}{l}2988 \\
13,60\end{array}$ & $\begin{array}{l}2827 \\
12,87\end{array}$ & $\begin{array}{r}1,548 \\
7,04\end{array}$ & $\begin{array}{r}1,405 \\
5,39\end{array}$ & $\begin{array}{r}634 \\
2,89\end{array}$ & $\begin{array}{l}29974 \\
100,00\end{array}$ \\
\hline Japäo & 1980 & $\stackrel{p}{p}$ & $\begin{array}{r}1577 \\
1,35\end{array}$ & $\begin{array}{r}5,987 \\
5,98\end{array}$ & $\begin{array}{r}18982 \\
16,24\end{array}$ & $\begin{array}{r}16,043 \\
13,72\end{array}$ & $\begin{array}{r}19859 \\
16,99\end{array}$ & $\begin{array}{r}17.537 \\
15.00\end{array}$ & $\begin{array}{r}1525 ! \\
13.04\end{array}$ & $\begin{array}{r}10,03 \\
8,64\end{array}$ & $\begin{array}{r}6.933 \\
5,93\end{array}$ & $\begin{array}{r}3646 \\
3,12\end{array}$ & $\begin{array}{r}116916 \\
100,00\end{array}$ \\
\hline Luxemburgo & 1978 & P & $\begin{array}{r}4 \\
1,12\end{array}$ & $\begin{array}{r}16 \\
4,48\end{array}$ & $\begin{array}{r}51 \\
14,29\end{array}$ & $\begin{array}{r}55 \\
15,41\end{array}$ & $\begin{array}{r}53 \\
14,85\end{array}$ & $\begin{array}{r}48 \\
13,45\end{array}$ & $\begin{array}{r}48 \\
13,45\end{array}$ & $\begin{array}{r}35 \\
9,80\end{array}$ & $\begin{array}{r}31 \\
8,68\end{array}$ & $\begin{array}{r}16 \\
4,48\end{array}$ & $\begin{array}{r}357 \\
100,00\end{array}$ \\
\hline México & 1975 & $\begin{array}{l}\mathrm{p} \\
s\end{array}$ & $\begin{array}{r}2353 \\
3,91\end{array}$ & $\begin{array}{l}8607 \\
14,31\end{array}$ & $\begin{array}{r}16916 \\
28,13\end{array}$ & $\begin{array}{r}11671 \\
19,40\end{array}$ & $\begin{array}{l}7676 \\
12,76\end{array}$ & $\begin{array}{l}5,271 \\
8,76\end{array}$ & $\begin{array}{r}3,515 \\
5,84\end{array}$ & $\begin{array}{r}2066 \\
3,44\end{array}$ & $\begin{array}{r}1375 \\
2,29\end{array}$ & $\begin{array}{r}696 \\
1,16\end{array}$ & $\begin{array}{l}60 \quad 145 \\
100,00\end{array}$ \\
\hline Noruega & 1980 & $\stackrel{P}{\%}$ & $\begin{array}{r}59 \\
1,25\end{array}$ & $\begin{array}{r}210 \\
5,14\end{array}$ & $\begin{array}{r}645 \\
15,79\end{array}$ & $\begin{array}{r}624 \\
15,27\end{array}$ & $\begin{array}{r}620 \\
15.17\end{array}$ & $\begin{array}{r}456 \\
11,16\end{array}$ & $\begin{array}{l}411 \\
10,06\end{array}$ & $\begin{array}{r}467 \\
11,43\end{array}$ & $\begin{array}{r}363 \\
8,88\end{array}$ & $\begin{array}{r}240 \\
5.37\end{array}$ & $\begin{array}{c}4086 \\
100,06\end{array}$ \\
\hline Hova Zelāndıa & 1979 & p & $\begin{array}{r}51 \\
1,63\end{array}$ & $\begin{array}{r}209 \\
6,69\end{array}$ & $\begin{array}{r}607 \\
19,43\end{array}$ & $\begin{array}{r}576 \\
18,44\end{array}$ & $\begin{array}{r}466 \\
14,92\end{array}$ & $\begin{array}{r}346 \\
11,08\end{array}$ & $\begin{array}{r}308 \\
9,86\end{array}$ & $\begin{array}{r}264 \\
8,45\end{array}$ & $\begin{array}{r}193 \\
6, \uparrow 8\end{array}$ & $\begin{array}{r}105 \\
3,36\end{array}$ & $\begin{array}{c}3124 \\
100,00\end{array}$ \\
\hline Panamä & 1980 & P & $\begin{array}{r}53 \\
2,88\end{array}$ & $\begin{array}{r}243 \\
13,21\end{array}$ & $\begin{array}{r}501 \\
27,23 \\
\end{array}$ & $\begin{array}{r}348 \\
18,91\end{array}$ & $\begin{array}{r}237 \\
12,88\end{array}$ & $\begin{array}{r}173 \\
9,40\end{array}$ & $\begin{array}{r}129 \\
7,01\end{array}$ & $\begin{array}{r}85 \\
4,62\end{array}$ & $\begin{array}{r}45 \\
2,45\end{array}$ & $\begin{array}{r}24 \\
1,30\end{array}$ & $\begin{array}{r}1840 \\
100,00\end{array}$ \\
\hline Polôn ia & 1979 & : & $\begin{array}{r}676 \\
1,91\end{array}$ & $\begin{array}{r}2,544 \\
7,18\end{array}$ & $\begin{array}{l}5330 \\
15,05\end{array}$ & $\begin{array}{l}6209 \\
17,53\end{array}$ & $\begin{array}{r}5965 \\
16.84\end{array}$ & $\begin{array}{l}3994 \\
11,28\end{array}$ & $\begin{array}{r}4286 \\
12,10\end{array}$ & $\begin{array}{r}2799 \\
7,90\end{array}$ & $\begin{array}{r}2411 \\
6,81\end{array}$ & $\begin{array}{r}1,199 \\
3,39\end{array}$ & $\begin{array}{l}35413 \\
100,00\end{array}$ \\
\hline Portugal & 1979 & p & $\begin{array}{r}180 \\
1,83\end{array}$ & $\begin{array}{r}710 \\
7,20\end{array}$ & $\begin{array}{r}1866 \\
18,92\end{array}$ & $\begin{array}{l}1662 \\
16,85\end{array}$ & $\begin{array}{r}266 \\
12,84\end{array}$ & $\begin{array}{r}1,183 \\
11,99\end{array}$ & $\begin{array}{l}1128 \\
11,44\end{array}$ & $\begin{array}{r}895 \\
9,07\end{array}$ & $\begin{array}{r}673 \\
6,82\end{array}$ & $\begin{array}{r}301 \\
3,05\end{array}$ & $\begin{array}{r}9863 \\
100,00\end{array}$ \\
\hline Romèn ia & 1980 & p & $\begin{array}{r}394 \\
1.77\end{array}$ & $\begin{array}{r}1,612 \\
7,26\end{array}$ & $\begin{array}{l}3916 \\
17,64\end{array}$ & $\begin{array}{l}3186 \\
14,35\end{array}$ & $\begin{array}{r}3261 \\
14,69\end{array}$ & $\begin{array}{l}2802 \\
12.62\end{array}$ & $\begin{array}{l}289 . \\
13,02\end{array}$ & $\begin{array}{r}1859 \\
8,37\end{array}$ & $\begin{array}{r}1,569 \\
7,07\end{array}$ & $\begin{array}{r}712 \\
3,21\end{array}$ & $\begin{array}{l}22201 \\
100,00\end{array}$ \\
\hline Singapura & 1980 & p & $\begin{array}{r}37 \\
1,53\end{array}$ & $\begin{array}{r}157 \\
6,50\end{array}$ & $\begin{array}{r}450 \\
19,06\end{array}$ & $\begin{array}{r}584 \\
24,19\end{array}$ & $\begin{array}{r}465 \\
19,26\end{array}$ & $\begin{array}{r}267 \\
\$ 1,06\end{array}$ & $\begin{array}{r}200 \\
8,29\end{array}$ & $\begin{array}{r}131 \\
5,43\end{array}$ & $\begin{array}{r}83 \\
3,44\end{array}$ & $\begin{array}{r}31 \\
1,28\end{array}$ & $\begin{array}{r}2414 \\
100,00\end{array}$ \\
\hline Suécia & 1980 & $\begin{array}{l}\mathrm{P} \\
q\end{array}$ & $\begin{array}{r}96 \\
1,16\end{array}$ & $\begin{array}{r}391 \\
4,70\end{array}$ & $\begin{array}{l}1141 \\
13,73\end{array}$ & $\begin{array}{r}125 \\
13,54\end{array}$ & $\begin{array}{r}251 \\
15,05\end{array}$ & $\begin{array}{r}1078 \\
12,97\end{array}$ & $\begin{array}{r}896 \\
10,78\end{array}$ & $\begin{array}{r}979 \\
11,78\end{array}$ & $\begin{array}{r}824 \\
9,91\end{array}$ & $\begin{array}{r}530 \\
6,38\end{array}$ & $\begin{array}{c}8311 \\
100,00\end{array}$ \\
\hline Sulca & 1980 & P & $\begin{array}{r}73 \\
1,16\end{array}$ & $\begin{array}{r}280 \\
4,43\end{array}$ & $\begin{array}{r}894 \\
14,16\end{array}$ & $\begin{array}{r}988 \\
15,65\end{array}$ & $\begin{array}{r}979 \\
15,51\end{array}$ & $\begin{array}{r}862 \\
13,65\end{array}$ & $\begin{array}{r}747 \\
11.83\end{array}$ & $\begin{array}{r}620 \\
9,82\end{array}$ & $\begin{array}{r}531 \\
8,41\end{array}$ & $\begin{array}{r}340 \\
5,38\end{array}$ & $\begin{array}{r}6314 \\
100,00\end{array}$ \\
\hline Trinidad e Tobago & 1977 & p & $\begin{array}{r}24 \\
2,14\end{array}$ & $\begin{array}{r}100 \\
8,94\end{array}$ & $\begin{array}{r}279 \\
24,93\end{array}$ & $\begin{array}{r}249 \\
22,25\end{array}$ & $\begin{array}{r}162 \\
14,48\end{array}$ & $\begin{array}{r}107 \\
9,56\end{array}$ & $\begin{array}{r}87 \\
7,77\end{array}$ & $\begin{array}{r}66 \\
5,90\end{array}$ & $\begin{array}{r}35 \\
3,93\end{array}$ & $\begin{array}{r}11 \\
0,98\end{array}$ & $\begin{array}{r}1119 \\
100,00\end{array}$ \\
\hline Urugua i & 1977 & $\begin{array}{l}p \\
*\end{array}$ & $\begin{array}{r}54 \\
1,90\end{array}$ & $\begin{array}{r}207 \\
3,27\end{array}$ & $\begin{array}{r}506 \\
17,78\end{array}$ & $\begin{array}{r}450 \\
15,81\end{array}$ & $\begin{array}{r}372 \\
13.07\end{array}$ & $\begin{array}{r}366 \\
12,86\end{array}$ & $\begin{array}{r}346 \\
12,16\end{array}$ & $\begin{array}{r}265 \\
9,31\end{array}$ & $\begin{array}{r}178 \\
6,25\end{array}$ & $\begin{array}{r}101 \\
3,55\end{array}$ & $\begin{array}{r}2846 \\
100,00\end{array}$ \\
\hline \multicolumn{3}{|c|}{ Mediana da de participacão } & 1,560 & 6,530 & 17,185 & 17,025 & 14,880 & 11,575 & 10.755 & 8,910 & 6,815 & 3,625 & \\
\hline
\end{tabular}

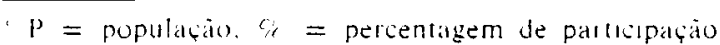


NOVO. N.F. et al. Ordenação de populações em amplas classes de nível de saúde, segundo um indicador abrangente definido por uma função discriminante linear. Rev. Saúde públ., S. Paulo, 19:344-63, 1985.

Padronização de coeficientes. População mediana

Sendo a idade, entre os atributos eventualmente associados com a variável estudada, a mais importante e mais frequientemente posta em foco, apenas ela foi considerada na padronização dos coeficientes.

Surgiu, então, como sempre ocorre em tal procedimento, o problema da escolha da população a ser adotada como padrão.

No presente trabalho, o problema da escolha do padrão foi consideravelmente dificultado, pois, como se vê na Tabela 1 , há acentuadas diferenças entre as populações no referente à participação dos grupos etários na sua composição.

\section{População padrão mediana}

Tendo em mente a recomendação de Shryock e Siegel ${ }^{7}$ (1973), salientando que a "regra geral é selecionar a distribuição de idades que seja semelhante à distribuição das várias populações estudadas", pareceu válida a idéia de definir uma "População Padrão Mediana".
Partindo das percentagens de participação dos grupos etários na composição da população de cada um dos países estudados, foi determinada a percentagem mediana para cada grupo etário. Essas medianas fornecem, então, a composição, em termos de percentagem de participação de cada grupo etário em uma população padrão. Visando a alcançar simplificação operacional, procedeu-se a ajustamento proporcional, para que a soma fosse igual a 100 ; finalmente, com arredondamento ao milhar, foram estabelecidas as frequiências de cada grupo em uma população igıal a um milhão.

A escolha da mediana das percentagens de participação, em lugar da média aritmética, visou a afastar a influência que, no caso desta, seria exercida pela eventual ocorrência, em certos grupos etários, de percentagens de participação fortemente discrepantes das demais.

Na Tabela 1 são também apresentados os dados básicos para o cálculo da "População Padrão Mediana" e, na Tabela 2 os países que delimitam a mediana, bem como os valores ajustados, ao milhar, para os grupos etários dessa população.

\section{TABELA 2}

Percentagem mediana de participação, países que a delimitam, percentagem ajustada e população padrão mediana, calculada em valores arredondados ao milhar, segundo os grupos etários

\begin{tabular}{cclrr}
\hline $\begin{array}{c}\text { Grupo } \\
\text { etário }\end{array}$ & $\begin{array}{c}\text { Percentagem } \\
\text { mediana }\end{array}$ & Países que delimitam a mediana & $\begin{array}{c}\text { Percentagem } \\
\text { ajustada }\end{array}$ & $\begin{array}{c}\text { População } \\
\text { padrão } \\
\text { mediana }\end{array}$ \\
\hline$<1$ & 1,560 & Barbados, Grécia e Singapura & 1,578 & 16.000 \\
$1-4$ & 6,530 & Irlanda do Norte e Singapura & 6,605 & 66.000 \\
$5-14$ & 17,185 & Austrália e Estados Unidos & 17,383 & 174.000 \\
$15-24$ & 17,025 & Irlanda e Portugal & 17,221 & 172.000 \\
$25-34$ & 14,880 & Bulgária e Luxemburgo & 15,052 & 150.000 \\
$35-44$ & 11,575 & Canadá e Escócia & 11,708 & 117.000 \\
$45-54$ & 10,755 & Hong Kong e Suécia & 10,879 & 109.000 \\
$55-64$ & $\mathbf{8 , 9 1 0}$ & Austrália e Irlanda & 9,013 & 90.000 \\
$65-74$ & 6,815 & Polônia e Portugal & 6,894 & 69.000 \\
75 e + & 3,625 & Finlândia e Uruguai & 3,667 & 37.000 \\
\hline Total & 98,860 & & 100,000 & 1.000 .000 \\
\hline
\end{tabular}


NOVO, N.F. et al. Ordenação de populações em amplas classes de nível de saúde, segundo um indicador abrangente definido por uma função discriminante linear. Rev. Saúde públ.. S. Paulo, 19:344-63, 1985.

Padronização dos coeficientes de mortalidade geral e de mortalidade por doenças transmissiveis

Uma vez obtida a "População Padrão Mediana", pôde-se calcular os coeficientes padronizados de mortalidade geral e de mortalidade por doenças transmissíveis, este com os valores corrigidos considerando os óbitos por causas mal definidas.

Foi adotado o método direto de padronização, por ser o que permite comparação dos valores obtidos para os diferentes países.

Esses coeficientes padronizados serão os utilizados em todos os cálculos e fórmulas, com as siglas CMDTp e CMGp.

\section{Cálculo das funções discriminantes lineares}

Para o cálculo das funções discriminantes, levando em conta que quatro dos seis indicadores sāo representados por taxas, resolveu-se, seguindo recomendação de Sokal e Rohlf ${ }^{y}$ (1969), adotar a transformação recíproca $\frac{1}{\mathrm{X}}$ dos valores de tais indicadores, ou seja, do CMI, do $\mathrm{CN}$, do CMDTp e do CMGp, mantendo os valores numéricos da $\mathrm{EV} e$ do IG. Para evitar decimais antes do primeiro algarismo significativo, as recíprocas foram calculadas pela razão 1000

valor numérico do indicador

Além disso,

para simplificar a expressão das equações em que figurem esses valores transformados e não transformados, convencionou-se adotar os seguintes símbolos: $\mathrm{X}_{1}=\frac{1000}{\mathrm{CMI}} ; \mathrm{X}_{2}=\frac{1000}{\mathrm{CN}} ; \mathrm{X}_{3}=\mathrm{EV}$; $\mathrm{X}_{4}=\frac{1000}{\mathrm{CMDTp}} ; \mathrm{x}_{5}=\frac{1000}{\mathrm{CMGp}}$ e $\mathrm{X}_{6}=$
IG. Foi essa transformação que proporcionou os maiores valores para a distância quadrática generalizada de Mahalanobis.

\section{Formação dos grupos de países a serem estudados}

A técnica da função discriminante tem sido aplicada em vários campos da biologia, especialmente em sistemática. Para a classificação dos integrantes de cada um dos grupos é usualmente possível utilizar critérios objetivos, mas no presente estudo, fundamento desta natureza não existe para essa classificação.

Swaroop e Uemura ${ }^{10}$ (1957) enfrentaram o mesmo problema, ou seja, o de constituir dois grupos de países que, do ponto de vista da saúde" fossem, ou "desenvolvidos" ou "subdesenvolvidos". Em seus próprios termos: "escolhemos deliberadamente dois grupos de países que sabemos a priori diferirem um do outro quanto a seus níveis de saúde".

Pareceu-nos conveniente basear nossa escolha dos países de cada grupo em algum critério menos subjetivo. Era necessário definir tal critério, capaz de permitir uma ordenação dos países, em termos de nível de saúde, avaliado, por alguma forma, com base nos indicadores escolhidos. Visando a afastar defeitos identificáveis em outras soluções possiveis (soma de postos nas ordenações de cada indicador; média de "notas" atribuídas a cada país, para cada indicador, por exemplo), adotou-se como variável, o desvio reduzido de cada valor $\mathrm{d}_{\mathrm{x}_{1}}$, contado a partir da média da distribuição a que pertence, ou seja:

$$
\begin{aligned}
& \mathrm{d}_{\mathrm{xi}}=\frac{\mathrm{Xi}-\overline{\mathrm{X}} \mathbf{i}}{\bar{\sigma}_{\mathrm{X} \mathbf{i}}}, \text { em que } \\
& \mathrm{Xi}=\begin{array}{l}
\mathrm{o} \text { valor numérico de indicador } \\
\text { não expresso em taxa (EV e IG) }
\end{array} \\
& \text { ou }
\end{aligned}
$$


NOVO, N.F. et al. Ordenação de populações em amplas classes de nível de saúde, segundo um indicador abrangente definido por uma função discriminante linear. Rev. Saúde públ., S. Paulo, 19:344-63, 1985.

\section{TABELA 3}

Valores numéricos dos indicadores das variáveis $\mathrm{X}_{1}$ a $\mathrm{X}_{\mathrm{f}}$ e dos desvios reduzidos $\mathrm{d} \mathrm{X}_{1}=\frac{\mathrm{Xi}_{\mathrm{i}}-\overline{\mathrm{X}}}{\hat{\sigma}_{\mathrm{Xi}}}$, sua soma dos postos e o posto por ela ocupado na ordenação decrescente dos valores, segundo os países estudados

\begin{tabular}{|c|c|c|c|c|c|c|c|c|c|c|c|}
\hline \multirow{2}{*}{ PAIS } & \multicolumn{3}{|c|}{$\begin{array}{c}\text { COEFICIENTE DE MORTALIDADE } \\
\text { INFANTIL }\end{array}$} & \multicolumn{3}{|c|}{ COEFICIENTE DE NATALIDADE } & \multicolumn{2}{|c|}{$\begin{array}{l}\text { ESPERANCA DE VIDA } \\
\text { AO NASCER }\end{array}$} & \multicolumn{3}{|c|}{$\begin{array}{l}\text { COEFICIENTE OE MORTALIDADE POR } \\
\text { DOENCAS TRANSMISSIVEIS PADRONIZADO }\end{array}$} \\
\hline & Valor & $x_{1}=\frac{1000}{\text { CRT }}$ & $d x_{1}$ & Velor & $x_{2}=\frac{1000}{C N}$ & $d x_{2}$ & $X_{3}=E V$ & $d x_{3}$ & Valor & $x_{4}=\frac{1000}{\operatorname{cmotp}}$ & $d x_{4}$ \\
\hline Alemanha Federal & 12,6 & 79,365 & 0,278 & 10,1 & 99.010 & 2,018 & 73.5 & 0,406 & 6.6 & 151,515 & 0,525 \\
\hline Austrälia & 10,7 & 93,458 & 0,664 & 15,4 & 64.935 & 0,202 & 74,6 & 0,657 & 3,9 & 256,410 & 1,879 \\
\hline Austria & 14,3 & 69,930 & 0,020 & 12,1 & 82,645 & 1,146 & 72,7 & 0,223 & 6,6 & 151,515 & 0.525 \\
\hline Barbados & 25,1 & 39,841 & $-0,803$ & 16,8 & 59,524 & $-0,087$ & 69,9 & $-0,417$ & 20,4 & 49,020 & $-0,797$ \\
\hline Bëlgica & 13,6 & 73,529 & 0,119 & 12,4 & 80,845 & 1,039 & 72,9 & 0,269 & 7.9 & 126,582 & 0,204 \\
\hline Bulgäria & 20,2 & 49,505 & $-0,539$ & 14,5 & 68,966 & 0,417 & 71,2 & $-0,120$ & 7,0 & 142,857 & 0,414 \\
\hline Canadä & 12,0 & 83,333 & 0,387 & 15,3 & 65,359 & 0.224 & 74.7 & 0,680 & 4,7 & 212,766 & 1,316 \\
\hline Chile & 36,6 & 27,322 & $-1,145$ & 21,5 & 46,512 & $-0,780$ & 68,9 & $-0,646$ & 51,9 & 19,268 & $-1,181$ \\
\hline Costa Rica & 22,1 & 45,249 & $-0,655$ & 32,0 & 31,250 & $-1,594$ & 73,3 & 0,360 & 24,0 & 41,667 & $-0,892$ \\
\hline Dinamarca & 8,4 & 119,048 & 1,364 & 11,2 & 89,286 & 1,500 & 74,3 & 0,588 & 3,5 & 285,714 & 2,257 \\
\hline Egito & 73.5 & 13,605 & $-1,520$ & 37,2 & 26,882 & $-1,827$ & 54,8 & $-3,868$ & 27,5 & 36,364 & $-0,961$ \\
\hline Escöeia & 11,2 & 89,286 & 0,550 & 13,5 & 74,074 & 0,689 & 72,3 & 0,131 & 4,7 & 212,766 & 1,316 \\
\hline Espanha & 15,1 & 66,225 & $-0,081$ & 17,1 & 58,480 & $-0,142$ & 74,4 & 0.611 & 15,3 & 65,359 & $-0,586$ \\
\hline Estados Unidos & 14,1 & 70,922 & 0,047 & 15,4 & 64,935 & 0,202 & 73,5 & 0,406 & 7,9 & 126,582 & 0,204 \\
\hline Finlandia & 7.7 & 129,870 & 1,660 & 13.5 & 74,074 & 0.689 & 73,2 & 0,337 & 9,3 & 107,527 & $-0,042$ \\
\hline franca & 10,7 & 93,458 & 0,664 & 13,8 & 72,464 & 0.603 & 74,7 & 0,680 & 12,9 & 77,519 & $-0,430$ \\
\hline Grécia & 18,7 & 53,476 & $-0,430$ & 15.7 & 63,694 & 0,136 & 75,6 & 0,886 & 7,8 & 128,205 & 0,225 \\
\hline Guatemala & 81,1 & 12,330 & $-1,555$ & 41,8 & 23,923 & $-1,984$ & 57,9 & $-3,159$ & 265,1 & 3,772 & $-1,381$ \\
\hline Guiana & 46,5 & 21,505 & $-1,304$ & 28,4 & 35,211 & $-1,383$ & 69,1 & $-0,600$ & 95,6 & 10,460 & $-1,295$ \\
\hline Holanda & 8,6 & 116,279 & 1,288 & 12,8 & 78,125 & 0,905 & 76,0 & 0,977 & 4,3 & 232,558 & 1,571 \\
\hline Hong Kong & 11,8 & 84,746 & 0,425 & 17,2 & 58,140 & $-0,160$ & 74,9 & 0,726 & 19,6 & 51,020 & $-0,772$ \\
\hline Hungria & 23,2 & 43.103 & $-0,714$ & 13,9 & 71,942 & 0,575 & 69,3 & $-0,554$ & 12,0 & 83,333 & $-0,354$ \\
\hline I thas Mauricias & 32,3 & 30,960 & $-1,046$ & 26,1 & 38,314 & $-1,217$ & 66.3 & $-1,240$ & 55,5 & 18,018 & $-1,197$ \\
\hline Inglatera e Gales & 12,0 & 83,333 & 0,387 & 13,3 & 75,188 & 0,748 & 73,8 & 0,474 & 3,9 & 256,410 & 1,879 \\
\hline Irlanda & 15,0 & 66,667 & $-0,069$ & 21,1 & 47,393 & $-0,733$ & 72,0 & 0,063 & 10,1 & 99,010 & $-0,152$ \\
\hline Irlanda do Norte & 15.9 & 62,893 & $-0,172$ & 17,1 & 58.480 & $-0,142$ & 71,6 & $-0,029$ & 6,1 & 163,934 & 0,686 \\
\hline is lândia & 7,7 & 129,870 & 1,660 & 19,8 & 50,505 & $-0,567$ & 77,0 & 1,205 & 4,9 & 204,082 & 1,204 \\
\hline Israet & 15.3 & 65,359 & $-0,105$ & 24,1 & 41,949 & $-1,048$ & 74,0 & 0,520 & 13,3 & 75,188 & $-0,460$ \\
\hline |täli & 17.1 & 58,480 & $-0,293$ & 12,6 & 79,365 & 0,971 & 74,1 & 0,543 & 6,3 & 158,730 & 0,619 \\
\hline lugos lávia & 33,8 & 29,586 & $-1,083$ & 17,4 & 57,471 & $-0,196$ & 70,3 & $-0,326$ & 22,3 & 44,843 & $-0,851$ \\
\hline Japāo & 7,5 & 133,333 & 1,754 & 13,6 & 73,529 & 0,660 & 76,4 & 1,068 & 11,1 & 90,090 & $-0,267$ \\
\hline Luxemburgo & 8,1 & 123,457 & 1,484 & 11,4 & 87,719 & 1,416 & 72,1 & 0,086 & 6.5 & 153,846 & 0,556 \\
\hline México & 49,7 & 20,121 & $-1,342$ & 37,5 & 26,667 & $-1,838$ & 64,7 & $-1,605$ & 135,5 & 7,380 & $-1,335$ \\
\hline Noruega & 8,1 & 123,457 & 1,484 & 12,5 & 80,000 & 1,005 & 75,9 & 0,954 & 6,2 & 161,290 & 0,652 \\
\hline Nova Zelândia & 12,8 & 78,125 & 0,244 & 16,6 & 60,241 & $-0,048$ & 73,1 & 0.314 & 5,7 & 175.439 & 0,834 \\
\hline Panamā & 22,0 & 45,455 & $-0,649$ & 28,3 & 35,336 & $-1,376$ & 69,7 & $-0,463$ & 43.7 & 22,883 & $-1,135$ \\
\hline Polônia' & 21,1 & 47.393 & $-0,596$ & 19,4 & 51,546 & $-0,512$ & 71,3 & $-0,097$ & 16,4 & 60,976 & $-0,643$ \\
\hline Portugal & 26,0 & 38,462 & $-0,841$ & 16,3 & 61,350 & 0.011 & 70,9 & $-0,189$ & 16,4 & 60,976 & $-0,643$ \\
\hline Romenia & 29,3 & 34,130 & $-0,959$ & 18,0 & 55,556 & $-0,298$ & 69,3 & $-0,554$ & 10,3 & 97,087 & $-0,177$ \\
\hline Singapura & 11,7 & 85,470 & 0,445 & 17,1 & 58,480 & $-0,142$ & 71,7 & $-0,006$ & 33,2 & 30,120 & $-1,041$ \\
\hline Suécila & 6.9 & 144,928 & 2,071 & 11,7 & 85,470 & 1,296 & 76,0 & 0.977 & 4,9 & 204.082 & 1,204 \\
\hline Suiça & 9,1 & 109,890 & 1,113 & 11,6 & 86,207 & 1,336 & 75,8 & 0,931 & 6,1 & 163,934 & 0,686 \\
\hline Trinldad e Tobago & 26,9 & 37,175 & $-0,876$ & 24,3 & 41,152 & $-1,066$ & 68,9 & $-0,646$ & 39.3 & 25.445 & $-1,102$ \\
\hline Urugua i & 48,5 & 20,619 & $-1,329$ & 20,4 & 49,020 & $-0,647$ & 69,3 & $-0,554$ & 34,9 & 28,653 & $-1,060$ \\
\hline Médi de Xi & $\cdot$ & 69,194 & - & - & 61,149 & - & 71,725 & - & - & 110,800 & - \\
\hline Desvio Padrão de $X i$ & - & 36,560 & - & - & 18.761 & - & 4,376 & - & - & 77,483 & $\cdot$ \\
\hline
\end{tabular}


NOVO, N.F. et al. Ordenação de populações em amplas classes de nível de saúde, segundo um indicador abrangente definido por uma função discriminante linear. Rev. Saúde públ., S. Paulo, 19:344-63, 1985.

IABELA 3 - (continuaço)

\begin{tabular}{|c|c|c|c|c|c|c|c|}
\hline \multirow{2}{*}{ PATS } & \multicolumn{3}{|c|}{$\begin{array}{c}\text { COEFICIENTE DE MORTALIDADE } \\
\text { GERAL PADRONIZADO }\end{array}$} & \multicolumn{2}{|c|}{ INDICADOR DE GUEDES } & \multirow{2}{*}{$\begin{array}{l}\text { SOMA DOS DESVIOS } \\
\mathrm{dx}_{\mathrm{i}}\end{array}$} & \multirow{2}{*}{ POSTOS } \\
\hline & Valor & $x_{5}=\frac{1000}{C A G D}$ & $d x_{5}$ & $x_{6}=16$ & $d x_{6}$ & & \\
\hline Alemenha Federal & 8,5 & 117,647 & 0,328 & 42,7 & 0,656 & 4,211 & 10 \\
\hline Austrälia & 7,9 & 126,582 & 0,755 & 39,6 & 0,414 & 4,571 & 8 \\
\hline Austria & 9,0 & 111,111 & 0,016 & 42,6 & 0,648 & 2,578 & 18 \\
\hline Barbados & 9,9 & 101,010 & $-0,466$ & 37,8 & 0,274 & $-2,296$ & 32 \\
\hline Bélgíca & 9,0 & 111,111 & 0,016 & 42,7 & 0,656 & 2.303 & 19 \\
\hline Bulgäria & 10.1 & 99,010 & $-0,562$ & 40,3 & 0,469 & 0,079 & 25 \\
\hline Canadã & 7,8 & 128,205 & 0,832 & 37,8 & 0,274 & 3,713 & 11 \\
\hline Chile & 10,4 & 96,154 & $-0,698$ & 23,2 & $-0,865$ & $-5,315$ & 38 \\
\hline Costa Rica & 8,3 & 120,482 & 0,463 & 16,7 & $-1,372$ & $-3,690$ & 35 \\
\hline Dinamarca & 8,3 & 120,482 & 0,463 & 43,4 & 0,710 & 6,882 & 2 \\
\hline Egito & 16,3 & 61,350 & $-2,359$ & 3,9 & $-2,371$ & $-12,906$ & 44 \\
\hline Escöcia & 9,7 & 103,093 & $-0,367$ & 43.8 & 0,742 & 3,061 & 14 \\
\hline Espanha & 7,9 & 126,582 & 0,755 & 39.5 & 0,406 & 0,963 & 23 \\
\hline Estados Unidos & 8,4 & 119,048 & 0,395 & 38,5 & 0,328 & 1,582 & 21 \\
\hline Finlândia & 8,8 & 113,636 & 0,137 & 40,7 & 0,500 & 3,281 & 13 \\
\hline França & 7,8 & 128,205 & 0,832 & 41,7 & 0,578 & 2,927 & 16 \\
\hline Grëcia & 7,2 & 138,889 & 1,342 & 41,2 & 0,539 & 2,698 & 17 \\
\hline Gua tema la & 11,4 & 87.719 & $-1,101$ & $-12,2$ & $-3,626$ & $-12,806$ & 43 \\
\hline Guiana & 14,5 & 68,966 & $-1,996$ & 13,0 & $-1,661$ & $-8,239$ & 41 \\
\hline Hol anda & 7,3 & 136,986 & 1,251 & 42,8 & 0,664 & 6,656 & 3 \\
\hline Hong Kong & 7,4 & 135,135 & 1,163 & 34,6 & 0,024 & 1,406 & 22 \\
\hline Hungria & 11,3 & 88,496 & $-1,064$ & 40,1 & 0,453 & $-1,658$ & 29 \\
\hline Il has mouriclas & 13,0 & 76,923 & $-1,616$ & 22,7 & $-0,904$ & $-7,220$ & 40 \\
\hline Inglaterra e Goles & 8,6 & 116,279 & 0,263 & 44,5 & 0,796 & 4,547 & 9 \\
\hline Irlanda & 10,1 & 99,010 & $-0,562$ & 41,5 & 0,562 & $-0,891$ & 27 \\
\hline Irlanda do Norte & 10,0 & 100,000 & $-0,514$ & 41,6 & 0,570 & 0,399 & 24 \\
\hline Is lândia & 6,6 & 151,515 & 1.945 & 40,0 & 0,445 & 5,892 & 6 \\
\hline Israel & 8,3 & 120,482 & 0,463 & 38,1 & 0,297 & $-0,333$ & 26 \\
\hline |tálía & 8,2 & 121,951 & 0,534 & 41,8 & 0,586 & 2,960 & 15 \\
\hline lugos lávia & 9,9 & 101,010 & $-0,466$ & 33,6 & $-0,054$ & $-2,976$ & 34 \\
\hline Japāo & 6,9 & 144,928 & 1,630 & 39,2 & 0,383 & 5,228 & 7 \\
\hline Luxemburgo & 9,9 & 101,010 & $-0,466$ & 42,3 & 0,625 & 3.701 & 12 \\
\hline México. & 11,5 & 86,957 & $-1,137$ & 1,2 & $-2,581$ & $-9,838$ & 42 \\
\hline Noruegs & 7,4 & 135,135 & 1,163 & 44,4 & 0,788 & 6,046 & 4 \\
\hline Nova Zelândia & 8,8 & 113,636 & 0,137 & 39,2 & 0,383 & 1,864 & 20 \\
\hline Panamä & 7,9 & 126,582 & 0.755 & 16,1 & $-1,419$ & $-4,287$ & 36 \\
\hline Polónia & 9,5 & 105,263 & $-0,263$ & 35,4 & 0,086 & $-2,025$ & 30 \\
\hline Portugal & 10,2 & 98,039 & $-0,608$ & 37,3 & 0,235 & $-2,305$ & 31 \\
\hline Romên ia & $10 ; 9$ & 91,743 & $-0,909$ & 35,5 & 0,094 & $-2,803$ & 33 \\
\hline Singapura & 9,8 & 102,041 & $-0,417$ & 32,7 & $-0,124$ & $-1,285$ & 28 \\
\hline Suécia & 7,4 & 135,135 & 1,163 & 44,7 & 0,812 & 7,523 & 1 \\
\hline Suiça & 7,3 & 136,986 & 1,251 & 42,8 & 0,664 & 5,981 & 5 \\
\hline Trinidad e Tobago & 13,8 & 72,464 & $-1,829$ & 27,9 & $-0,499$ & $-6,018$ & 39 \\
\hline Urugua i & 10,3 & 97.087 & $-0,653$ & 32,0 & $-0,179$ & $-4,422$ & 37 \\
\hline $\begin{array}{l}\text { Média de Xi } \\
\text { Desvio Padrāo de Xi }\end{array}$ & $\begin{array}{l}- \\
-\end{array}$ & $\begin{array}{r}110,774 \\
20,947\end{array}$ & - & $\begin{array}{l}34,293 \\
12,821\end{array}$ & 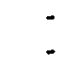 & $\begin{array}{l}- \\
-\end{array}$ & - \\
\hline
\end{tabular}


NOVO, N.F. et al. Ordenação de populações em amplas classes de nível de saúde, segundo um indicador abrangente definido por uma função discriminante linear. Rev. Saúde públ., S. Paulo, 19:344-63, 1985.

$$
\begin{aligned}
\overline{\mathrm{X}}_{\mathrm{i}} & =\frac{1000}{\begin{array}{l}
\text { valor numérico de indicador expresso } \\
\text { em taxa (CMI, CN, CMDTp e CMGp) }
\end{array}} \\
\mathrm{Xi} & =\text { média aritmética de Xi } \\
\bar{\sigma}_{\mathrm{X} i} & =\text { desvio padrão estimado de Xi }
\end{aligned}
$$

Definiu-se, então, como critério, o valor da soma algébrica, para cada país, dos desvios reduzidos calculados para cada indicador. Tal valor poderia, eventualmente, ser considerado um indicador abrangente dos indicadores incluídos no seu cálculo. Entretanto, isso implicaria admissão de pesos iguais para todos esses indicadores, hipótese que parece pouco aceitável. Além disso, sua determinação ficaria extremamente penosa quando se tratasse de país não incluído na relação, exigindo cálculo de novas médias, desvios padrão e desvios reduzidos para o novo grupo. Em última análise, o objetivo do presente estudo foi, exatamente, o de procurar estabelecer pesos que traduzissem a participação de cada indicador na composição de uma estatística capaz de permitir, como o fizeram Swaroop e Uemura ${ }^{10}$ (1957), a ordenação de países em amplas classes de nível de saúde.

Os valores das variáveis $\mathrm{X}_{1}$ a $\mathrm{X}_{6}$ e dos desvios reduzidos referentes a cada uma delas figuram na Tabela 3.

Tratava-se, em seguida, de definir o tamanho dos grupos de países a serem confrontados, no cálculo das funções discriminantes. Foi adotado o critério estabelecido por Kelley ${ }^{5}$ (1939) para a técnica de análise por ítens, de testes educacionais, atribuindo a cada grupo $27 \%$ do total de provas, em um e outro extremo da distribuição de resultados. Assim, resultaram dois grupos de tamanho 12, integrados por países com valores correspondentes aos dois extremos da distribuição da soma de desvios reduzidos.

Foram designados por "mais desenvolvidos" os 12 países com maior soma de desvios reduzidos. São eles: Alemanha Federal, Austrália, Canadá, Dinamarca, Holanda, Inglaterra e Gales, Islândia, Japão, Luxemburgo, Noruega, Suécia e Suiça. No grupo a ser com ele confrontado, o dos "menos desenvolvidos", figuram os 12 com menores somas, a saber: Chile, Costa Rica, Egito, Guatemala, Guiana, Ilhas Maurícias, Iugoslávia, México, Panamá, Romênia, Trinidad e Togabo e Uruguai.

\section{MÉTODOS ESTATISTICOS}

\section{Cálculo da função discriminante}

Como referem Snedecor e Cochran 8 (1971), em discussão sucinta, mas esclarecedora, sobre função discriminante, esta foi desenvolvida independentemente por Fisher, Mahalanobis e Hotteling, na década de 30. Para o cálculo dessa função, seguiu-se o modelo apresentado por Goulden ${ }^{3}$ (1952).

Foi utilizada a técnica passo a passo (stepwise), ascendente, como descrita por Armitage ${ }^{1}$ (1971). Calculou-se, inicialmente, o poder de discriminação de cada um dos indicadores, isoladamente. Esse poder de discriminação foi medido pela diferença quadrática de Mahalanobis. Depois de eleito o indicador único com maior poder de discriminação, foram acrescentados um a um os indicadores restantes, passando assim a trabalhar com funções com dois indicadores. Após escolher o par de indicadores com maior poder de discriminação, foram acrescentados cada um dos restantes, novamente selecionando o conjunto mais discriminador. Usando o mesmo procedimento, chegou-se às funções discriminantes com números crescentes de indicadores, mesmo que $o$ acréscimo não mais proporcionasse ganho significante na discriminação.

$O$ efeito da adição de cada indicador foi medido pela diferença observada entre as Somas de Quadrados, entre grupos, 
NOVO, N.F. et al. Ordenação de populações em amplas classes de nível de saúde, segundo um indicador abrangente definido por uma função discriminante linear. Rev. Saúde públ., S. Paulo, 19:344-63, 1985.

com o novo indicador e sem o mesmo. Essa diferença, dividida pelo Quadrado Médio do Resíduo da Análise de Variância (com o novo indicador incluído), forneceu a significância do ganho observado com a adição do referido indicador.

\section{Outros métodos estatísticos}

Além desses métodos estatísticos, foi utilizado, para os valores de $\mathrm{Z}$ obtidos nas equações discriminantes, o teste de aderência à curva normal, de Kolmogorov-Smirnov (Sokal e Rohlf ${ }^{\theta}, 1969$ ). A significância das estatísticas $G_{1}$ e $G_{2}$, para as mesmas distribuições de $Z$ foi determinada mediante teste " $t$ " de "Student". Decidiu-se rejeitar a hipótese de nulidade quando $\alpha \leqslant 0,05$.

\section{RESULTADOS}

Como já foi referido, a seleção das equações, envolvendo os seis indicadores utilizados no trabalho, baseou-se nos resultados do cálculo das funções discriminantes lineares. Essa seleção se fez pela aplicação da técnica passo a passo ascendente.

Inicialmente, o cálculo foi feito a partir dos valores numéricos dos indicadores. Tendo em vista que os maiores valores da diferença quadrática de Mahalanobis foram observados quando se trabalhou com as recíprocas dos valores numéricos dos indicadores representados por taxas, resolveu-se trabalhar com os valores de $X_{1}$ relacionados na Tabela 3 , ou seja, as recíprocas de $\mathrm{CMI}, \mathrm{CN}$, CMDTp e CMGp e os valores numéricos de EV e IG. Foram, assim, obtidos os valores apresentados na Tabela 4 que mostra dados complementares referentes a características das distribuições e às correlações entre elas. Observa-se, nessa tabela, que acréscimos significantes da soma de quadrados ocorrem até o quinto passo. Nesta tabela, na apresentação das equações discriminantes, selecionadas em cada passo, a partir do segundo, foi feita, também, a indicação da forma simplifi- cada que assumem pela divisão de todos os coeficientes pelo correspondente a $\mathrm{X}_{4}$.

\section{DISCUSSĀO}

Como se observa na Tabela 4, para fins de discriminação entre os dois grupos, de "mais desenvolvidos" e "menos desenvolvidos", a equação correspondente ao quinto passo deveria ser seleciona$\mathrm{da}$, pois, até ele, acréscimos significantes na soma de quadrados são proporcionados de passo a passo.

Entretanto, como foi apontado, nosso objetivo era o de selecionar a equação que mais adequadamente propiciasse a ordenação dos países em classes, em termos de nível de saúde.

Foi necessário, então, comparar as distribuições dos valores de $Z$, calculados segundo as equações selecionadas a cada passo, estudando o seu relacionamento com algum critério que se possa considerar válido.

Para tanto, foi inicialmente elaborada a Tabela 5 em que se encontram os valores de $\mathrm{Z}$ calculados nos termos dessas seis equações para os 44 países.

Desde logo cabe assinalar que, como se vê pelos valores fornecidos pelo teste de Kolmogorov-Smirnov e pelas estatísticas $G_{1}$ e $G_{2}$, nenhuma das distribuições de valores de $\mathbf{Z}$ afasta-se significantemente da normalidade.

Assume especial importância a ocorrência, nas equações que incluem mais de três indicadores, de sinais de coeficientes que não se coadunam com a lógica do problema. De fato, nas equações 4,5 e 6 , o sinal negativo de $\mathrm{X}_{6}$ implica tanto maior redução do valor de $\mathrm{Z}$ quanto maior o valor do Indicador de Guedes; também na equação 6 o sinal negativo de $X_{3}$ acarreta redução maior do valor de $\mathrm{Z}$ quando a Esperança de Vida aumenta.

Tal situação surge, com certa frequiência, em análises multivariadas, não só no 
NOVO, N.F. et al. Ordenação de populações em amplas classes de nível de saúde, segundo um indicador abrangente definido por uma função discriminante linear. Rev. Saúde públ., S. Paulo, 19:344-63, 1985.

\section{TABELA 4}

Desenvolvimento do cálculo das funções discriminantes pelo método passo a passo ascendente, a partir das recíprocas (x 1000), de CMI, CN, CMDTp e CMGp e dos valores numéricos de EV e IG. Características das distribuiçôes dessas variáveis nos grupos "mais desenvolvidos" e "menos desenvolvidos" e correlações entre elas, no conjunto desses grupos

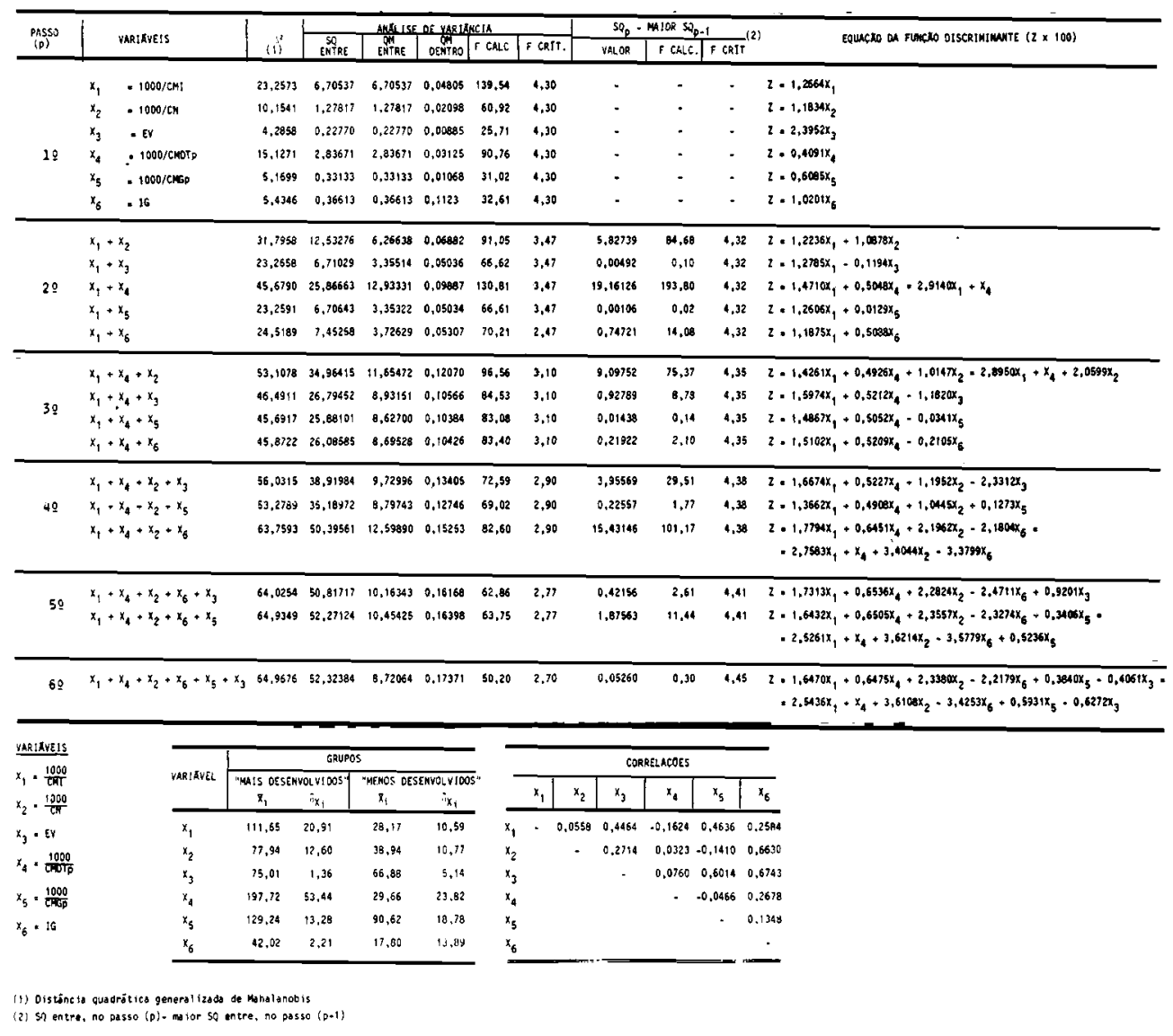


NOVO, N.F. et al. Ordenação de populações em amplas classes de nível de saúde, segundo um indicador abrangente definido por uma função discriminante linear. Rev. Saúde públ., S. Paulo, 19:344-63, 1985.

caso de funções discriminantes como também em regressões múltiplas, como resultado das complexas inter-relações entre as variáveis. Certamente não há interferência na capacidade discriminante, como comprovam os valores de $\Delta^{2}$, ou seja, mesmo com sinais que divergem do esperado, reduz-se a probabilidade de erro de classificação, de cada indivíduo estudado, em um ou outro dos grupos.

Para o presente objetivo, porém, impunha-se o exame da possibilidade de alteração da posição de países nas classes da ordenação a ser definida, especialmente para aqueles que apresentam valores de $\mathrm{EV}$ ou de IG grandemente discrepantes dos demais. Como exemplo frisante, basta referir o caso da Guatemala. Como se pode ver na Tabela 3, sua situação é desfavorável, em relação a todos os demais países, quanto aos valores dos indicadores CMI, CN, CMDTp e IG; entretanto, como mostra a Tabela 5 , seu valor de $Z$, calculado segundo a equação que inclui esses indicadores, supera os de cinco países. Ainda mais, com situação melhor do que a de cinco países, somente no que se refere ao CMGp, e apenas a de um desses países, no caso da $\mathrm{EV}$, seu valor de $\mathrm{Z}$ é maior do que o de seis outros quando calculado pela equação 6 .

Considerando todos esses aspectos, tornou-se necessário escolher um critério que permitisse selecionar, dentre as seis equações, a que apresente com ele maior relacionamento. Como foi referido, a soma dos desvios reduzidos, contados a partir das médias das variáveis $\mathrm{X}_{1}$ a $\mathrm{X}_{6}$, poderia constituir indicador razoavelmente expressivo, ainda que implique admissão de pesos iguais para todos os indicadores que abrange. $O$ relacionamento da sua distribuição com as dos valores de $\mathbf{Z}$, fornecido pelas seis equações, pode representar forma válida de se aferir o grau de adequação de cada uma delas ao fim visado. Com isso, pode-se avaliar até que ponto a presença de sinais discrepantes, em três dessas equações, afeta essa adequação.

Calculando as correlações entre os valores das somas de desvios reduzidos que figuram na Tabela 3 e cada uma das distribuições de valores de $Z$, referentes às seis equações discriminantes, arrolados na Tabela 5, foram encontrados para o coeficiente de correlação de Pearson ( $r$ ) e para o coeficiente de determinação $\left(r^{2}\right)$, os seguintes valores:

$\begin{array}{lcc}\text { Correlação com: } & r & r^{2} \\ Z=1,2664 X_{1} & 0,803 & 0,645 \\ Z=2,9140 X_{1}+X_{4} & 0,850 & 0,723 \\ Z=2,8950 X_{1}+2,0599 X_{2}+X_{4} & 0,858 & 0,736 \\ Z=2,7583 X_{1}+3,4044 X_{2}+X_{4}-3,3799 X_{6} & 0,824 & 0,678 \\ Z=2,5261 X_{1}+3,6214 X_{2}+X_{4}+0,5236 X_{5}-3,5779 X_{6} & 0,822 & 0,675 \\ Z=2,5436 X_{1}+3,6108 X_{2}-0,6272 X_{3}+X_{4}+0,5931 X_{5}-3,4253 X_{6} & 0,829 & 0,687\end{array}$

Sendo o valor crítico de $r$, ao nível de 0,05 , igual a 0,29 , todos os valores encontrados são significantes, correspondendo o maior à equação que inclui recíprocas de três indicadores, com sinais de coeficientes que são os de se prever em termos do comportamento das variáveis.
Lembrando que, como foi salientado, a soma dos desvios reduzidos implica a admissão de pesos iguais para os seis indicadores que dela participam, poderse-ia supor que o mesmo ocorresse no caso da equação discriminante para o qual foi encontrado o maior valor de $r$, ou seja, que os três indicadores parti- 
NOVO, N.F. et al. Ordenação de populações em amplas classes de nível de saúde, segundo um indicador abrangente definido por uma função discriminante linear. Rev. Saúde públ., S. Paulo, 19:344-63, 1985.

\section{TABELA 5}

Valores de $Z$, calculados nos termos das equações discriminantes calculadas em cada passo, segundo os países estudados. Média e desvio padrão. Teste de Kolmogorov-Smirnov e estatísticas $G_{1}$ e $G_{2}$ e respectivos testes $t$

\begin{tabular}{|c|c|c|c|c|c|c|}
\hline paIs & \begin{tabular}{c|c} 
& $(1)$ \\
ANO & $z=1,2664 x_{\text {, }}$
\end{tabular} & \begin{tabular}{|c|} 
(2) \\
$z=2,9140 x_{1}+x_{4}$
\end{tabular} & $\begin{array}{c}(3) \\
z=2,8950 x_{1}+2,0599 x_{2}+x_{4}\end{array}$ & $\begin{array}{c}(4) \\
z=2,7583 x^{+3}+3,4044 x_{2}+ \\
+x_{4}-3,3799 x_{6}\end{array}$ & $\begin{array}{c}(5) \\
2=2,5261 x_{1}+3,6214 x_{2}+x_{4}+ \\
+0,5236 x_{5}-3,5779 x_{6}\end{array}$ & 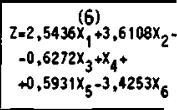 \\
\hline 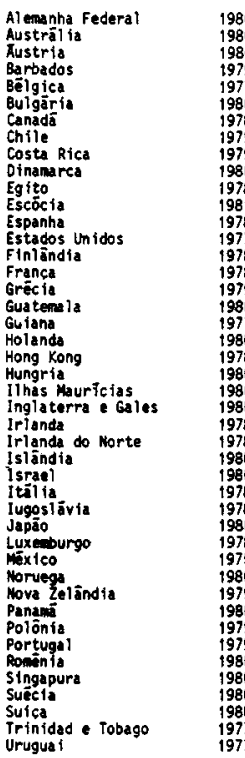 & 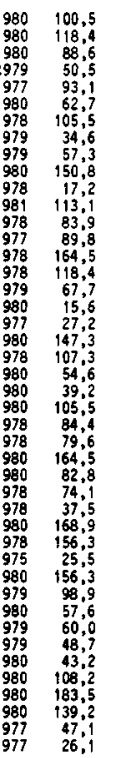 & 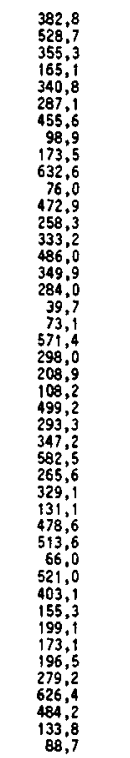 & 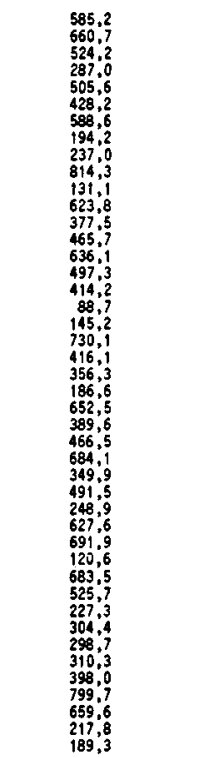 & 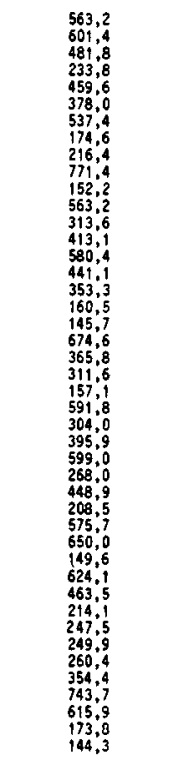 & 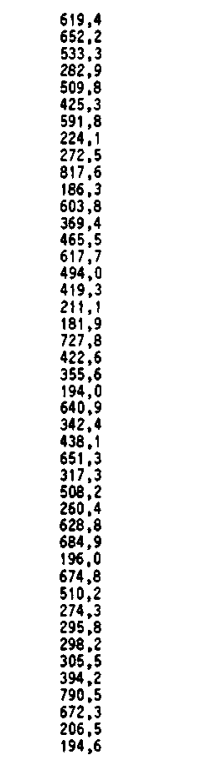 & 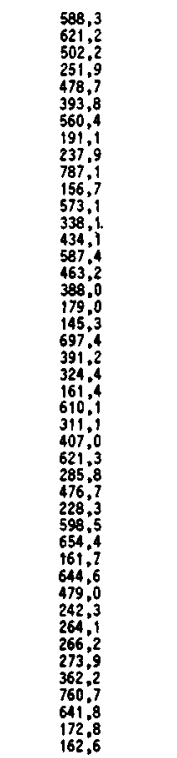 \\
\hline 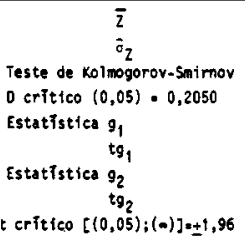 & $\begin{array}{l}87,6 \\
46,3 \\
0,0914 \\
0,3452 \\
0,97 \\
-0,9283 \\
-1,32\end{array}$ & $\begin{array}{l}312,4 \\
169,8 \\
0,0926 \\
0,1992 \\
0,050 \\
0,1,1251 \\
-1,60\end{array}$ & $\begin{array}{c}437.1 \\
200.3 \\
0,0779 \\
0.00472 \\
0.13 \\
-1,1882 \\
-1,61\end{array}$ & $\begin{array}{l}393,9 \\
185,4 \\
0,1146 \\
0,2535 \\
0,71 \\
-1,1983 \\
-1,11\end{array}$ & $\begin{array}{l}442,3 \\
186,7 \\
0,1116 \\
0,2212 \\
0,62 \\
-1,2087 \\
-1,72\end{array}$ & $\begin{array}{l}410,8 \\
187,6 \\
0,1106 \\
0,2182 \\
0,61 \\
-1,2087 \\
-1,72\end{array}$ \\
\hline
\end{tabular}

cipassem igualmente na discriminação entre os grupos.

A simples comparação dos valores dos coeficientes de cada uma das variáveis não permite avaliar essa participação, pois cada um deles deve ter valor tal que, de sua integração no conjunto resultem valores de $Z$, para cada um dos componentes dos dois grupos, $\mathbf{A}$ e $\mathbf{B}$, que assegurem a maximização da razão:
$\Delta^{2}=\frac{(\overline{\mathbf{Z}} \mathrm{A}-\overline{\mathbf{Z}} \mathrm{B})^{2}}{\text { variància estimada de } \mathrm{Z} \text { dentro de grupos }}$

Uma estimativa da participação de cada variável, na discriminação entre os grupos, pode ser feita em termos das quantidades com que contribuem para a diferença $\overline{\mathbf{Z}} \mathbf{A}-\overline{\mathbf{Z}} \mathbf{B}$. 
NOVO, N.F. et al. Ordenação de populações em amplas classes de nível de saúde, segundo um indicador abrangente definido por uma função discriminante linear. Rev. Saúde públ., S. Paulo, 19:344-63, 1985.

Com esse objetivo, foi elaborada inicialmente a Tabela 6, em que se encontram, destacados na Tabela 5, os valores de $Z$, calculados segundo a equação que inclui as variáveis $\mathrm{X}_{1}, \mathrm{X}_{2}$ e $\mathrm{X}_{4}$, corres- pondentes aos países "mais desenvolvidos" e "menos desenvolvidos", bem como, destacados da Tabela 3, os valores de $X_{1}, X_{2}$ e $X_{4}$ para esses países.

\section{TABELA 6}

Valores de $Z=2,8950 X_{1}+2,0599 X_{2}+X_{4}$ e valores de $X_{1}, X_{2}$ e $X_{4}$, em países "mais desenvolvidos" e "menos desenvolvidos"

\begin{tabular}{lrrrr}
\hline & \multicolumn{4}{c}{ "Menos desenvolvidos" } \\
\cline { 2 - 5 } Países & $\mathrm{X}_{1}$ & $\mathrm{X}_{2}$ & $\mathrm{X}_{4}$ & $\mathrm{Z}$ \\
\hline Dinamarca & 119,048 & 89,286 & 285,714 & 814,3 \\
Suécia & 144,928 & 85,470 & 204,082 & 799,7 \\
Holanda & 116,279 & 78,125 & 232,558 & 730,1 \\
Luxemburgo & 123,457 & 87,719 & 153,846 & 691,9 \\
Islândia & 129,870 & 50,505 & 204,082 & 684,1 \\
Noruega & 123,457 & 80,000 & 161,290 & 683,5 \\
Austrália & 93,458 & 64,935 & 256,410 & 660,7 \\
Suiça & 109,890 & 86,207 & 163,934 & 659,6 \\
Inglaterra e Gales & 83,333 & 75,188 & 256,410 & 652,5 \\
Japão & 133,333 & 73,529 & 90,090 & 627,6 \\
Canadá & 83,333 & 65,359 & 212,766 & 588,6 \\
Alemanha & 79,365 & 99,010 & 151,515 & 585,2 \\
\hline \multicolumn{1}{c}{ Média } & 111,646 & 77,944 & 197,725 & 681,48 \\
\hline
\end{tabular}

\begin{tabular}{|c|c|c|c|c|}
\hline \multirow[b]{2}{*}{ Países } & \multicolumn{4}{|c|}{ "Mais desenvolvidos" } \\
\hline & $x_{1}$ & $\mathrm{X}_{2}$ & $X_{4}$ & $\mathrm{Z}$ \\
\hline Guatemala & 12,330 & 23,923 & 3,772 & 88,7 \\
\hline México & 20,121 & 26,667 & 7,380 & 120,6 \\
\hline Egito & 13,605 & 26,882 & 36,364 & 131,1 \\
\hline Guiana & 21,505 & 35,211 & 10,460 & 145,2 \\
\hline Ilhas Maurícias & 30,960 & 38,314 & 18,018 & 186,6 \\
\hline Uruguai & 20,619 & 49,020 & 28,653 & 189,3 \\
\hline Chile & 27,322 & 46,512 & 19,268 & 194,2 \\
\hline Trinidad e Tobago & 37,175 & 41,152 & 25,445 & 217,8 \\
\hline Panamá & 45,455 & 35,336 & 22,883 & 227,3 \\
\hline Costa Rica & 45,249 & 31,250 & 41,667 & 237,0 \\
\hline Iugoslávia & 29,586 & 57,471 & 44,843 & 248,9 \\
\hline Romênia & 34,130 & 55,556 & 97,087 & 310,3 \\
\hline Média & 28,171 & 38,941 & 29,653 & 191,42 \\
\hline
\end{tabular}


NOVO, N.F. et al. Ordenação de populações em amplas classes de nível de saúde, segundo um indicador abrangente definido por uma função discriminante linear. Rev. Saúde públ., S. Paulo, 19:344-63, 1985.

Calculando as equações com os valores médios de $\mathrm{X}_{1}, \mathrm{X}_{2}$ e $\mathrm{X}_{4}$ para cada um dos grupos, tem-se:

$$
\text { "mais desenvolvidos": } \begin{aligned}
\overline{\mathrm{Z}}_{\mathrm{A}} & =2,8950 \times 111,646+2,0599 \times 77,944+197,725= \\
& =323,215+160,557+197,725=681,497 \\
& \\
\text { "menos desenvolvidos": } \overline{\mathrm{Z}}_{\mathrm{B}} & =2,8950 \times 28,171+2,0599 \times 38,941+29,653= \\
& =81,555+80,215+29,653=191,423
\end{aligned}
$$

Subtraindo, membro a membro, a equação (2) da equação (1), tem-se:

$$
\overline{\mathrm{Z}}_{\mathrm{A}}-\overline{\mathrm{Z}}_{\mathrm{B}}=241,660+80,342+168,072=490,074
$$

Então, para a composição da diferença entre $\bar{Z}_{\mathrm{A}}$ e $\overline{\mathrm{Z}}_{\mathrm{B}}, \mathrm{X}_{1}$ concorre com $49,3 \%, X_{2} \operatorname{com} 16,4 \%$ e $X_{4} \operatorname{com} 34,3 \%$, ou seja, diferem os pesos com que participam na discriminação os três indicadores. Considerando este fato e lembrando que a soma dos desvios reduzidos implica pesos iguais para todas as variáveis, torna-se compreensível não ter sido encontrada correlação perfeita entre os valores de $\mathrm{Z}$ e as somas de desvios reduzidos.

Ainda visando à comparação entre as seis equações, procedeu-se à ordenação em classes dos valores de $Z$, decorrentes de cada uma delas, para os 44 países estudados.

Não podendo ser rejeitada, como já foi referido, a hipótese de normalidade para todas essas distribuições de $Z$, pôde-se organizar classes a partir das respectivas médias, adotando-se, como in$\bar{\sigma} \mathbf{z}$

tervalo, $\frac{-}{2}$, todos os valores com aproximação à unidade. Estes parâmetros estão arrolados na Tabela 5. As classes, seguindo a ordem decrescente dos valores de $Z$, são designadas, em maiúsculas, em ordem alfabética.

$\mathrm{Na}$ Tabela 7 figuram os resultados dessas ordenações em classes. Como se pode ver, a classe $\mathrm{H}$ deixa de existir, nas ordenações baseadas em equações incluindo quatro ou mais indicadores, como consequiência das alterações introduzidas no valor de $Z$, capazes de influir na ordenação dos países quando os valores de IG ou de EV, ou de ambos, discrepam acentuadamente dos demais. Tais discrepâncias ocorrem, justamente, na cauda inferior das distribuições desses dois indicadores como se pode observar na Tabela 3.

Assim, o sinal negativo nos coeficientes dessas duas variáveis provoca uma mudança de classe, para melhor, dos países que apresentam situação mais desfavorável em termos desses dois indicadores.

Lembrando que a equação com três recíprocas de indicadores foi a que proporcionou valores de $\mathrm{Z}$ com a maior correlação com a soma dos desvios reduzidos, ainda que sem diferenças significantes entre os valores dos coeficientes, as considerações acima aduzem mais um argumento em favor de sua escolha como a mais adequada para o objetivo visado neste estudo.

Não é ela, como mostra a Tabela 4 , a que corresponde ao maior valor de $\Delta^{2}$ na técnica passo a passo, mas, como já foi referido, não só esse valor foi considerado, tendo-se atentado primordialmente na adequação para fins de ordenação. De qualquer forma, mesmo em termos de discriminação, o valor 7,29 alcançado por $\Delta$, é plenamente satisfatório. 
NOVO, N.F, et al. Ordenação de populações em amplas classes de nível de saúde, segundo um indicador abrangente definido por uma função discriminante linear. Rev. Saúde públ., S. Paulo, 19:344-63, 1985.

\section{TABELA 7}

Países, segundo a classificação nas ordenações baseadas nos valores de $\mathbf{Z}$ calculados em termos das seis equações discriminantes

\begin{tabular}{|c|c|c|c|c|c|c|c|c|}
\hline \multirow{2}{*}{ EOUACOES } & \multicolumn{8}{|c|}{ CLASSES E RESPECTIYOS LIMITES } \\
\hline & $\mathrm{H}$ & G & $\mathbf{F}$ & $\mathbf{E}$ & D & $\mathrm{C}$ & B & A \\
\hline$Z=1,2664 x_{1}$ & $\begin{array}{l}-19 \\
\text { Egito } \\
\text { Gua temala }\end{array}$ & $\begin{array}{l}\quad 19-42 \\
\text { Chile } \\
\text { Guiana } \\
\text { linas Mauricias } \\
\text { Iugoslavia } \\
\text { Mexico } \\
\text { Urugua }\end{array}$ & \begin{tabular}{|l}
\multicolumn{1}{|c|}{$42 \curvearrowleft 65$} \\
Barbados \\
Bulgária \\
Costa Rica \\
Hungria \\
Panamá \\
Polónia \\
Portugal \\
Roménia \\
Trinidad e Tobago
\end{tabular} & $\begin{array}{l}\quad 65 \leftarrow 88 \\
\text { Espanta } \\
\text { Grocta } \\
\text { Irlanda } \\
\text { Irlanda do Norte } \\
\text { Israel } \\
\text { Itália }\end{array}$ & 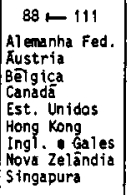 & $\begin{array}{l}111 \vdash 134 \\
\text { Austräll ia } \\
\text { Escócia } \\
\text { Franca }\end{array}$ & $\begin{array}{l}134 \text { D- } 157 \\
\text { Dinanarea } \\
\text { Holanda } \\
\text { Luxenburgo } \\
\text { Noruega } \\
\text { Sutica }\end{array}$ & $\begin{array}{l}157 \text { - } \\
\text { Finländta } \\
\text { Isländia } \\
\text { Japäo } \\
\text { Suếcia }\end{array}$ \\
\hline$z=2,9140 x_{1}+x_{4}$ & $\begin{array}{c}-57 \\
\text { Gua temala }\end{array}$ & $\begin{array}{l}\quad 57-142 \\
\text { Chile } \\
\text { Egito } \\
\text { Gianana } \\
\text { Ulhas Mauricias } \\
\text { Iugoslävia } \\
\text { Mexico } \\
\text { Trinidad - Tobago } \\
\text { Uruguai }\end{array}$ & $\begin{array}{l}142 \vdash 227 \\
\text { Barbados } \\
\text { Costa Rica } \\
\text { Hungria } \\
\text { Panamá } \\
\text { Polónia } \\
\text { Portugat } \\
\text { Romentia }\end{array}$ & $\begin{array}{l}227-312 \\
\text { Bulgäria } \\
\text { Espanha } \\
\text { Grecta } \\
\text { Hong Kong } \\
\text { Irlanda } \\
\text { Itrael } \\
\text { Singapura }\end{array}$ & 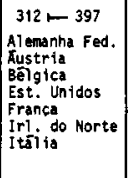 & $\begin{array}{l}397 \longmapsto 482 \\
\text { Canadä } \\
\text { Escōcta } \\
\text { Japāa } \\
\text { N.Zu ầndia }\end{array}$ & $\begin{array}{l}482 \curvearrowleft 567 \\
\text { Austrälia } \\
\text { Finiandia } \\
\text { Ingl.e Gales } \\
\text { Luxemburgo } \\
\text { Noruega } \\
\text { Suica }\end{array}$ & $\begin{array}{l}567 \vdash \\
\text { Dinanarca } \\
\text { Holanda } \\
\text { Isländia } \\
\text { Suêcia }\end{array}$ \\
\hline$z=2,8950 x_{1}+2,0599 x_{2}+x_{4}$ & $\begin{array}{l}-137 \\
\text { Egito } \\
\text { Guatemala } \\
\text { Mexico }\end{array}$ & $\begin{array}{l}137 \vdash 237 \\
\text { Chile } \\
\text { Guana } \\
\text { IIhas Maurictas } \\
\text { Panama } \\
\text { Trinidad e Tobago } \\
\text { Urugua } 1\end{array}$ & $\begin{array}{l}237 \vdash 337 \\
\text { Barbados } \\
\text { Costa Rica } \\
\text { Iugostâivia } \\
\text { Polonia } \\
\text { Portugal } \\
\text { Romenia }\end{array}$ & $\begin{array}{l}\text { 337 - } 437 \\
\text { Butgäria } \\
\text { Espanha } \\
\text { Grectia } \\
\text { Hong Yong } \\
\text { Hungria } \\
\text { Irranda } \\
\text { Israel } \\
\text { Singapura }\end{array}$ & \begin{tabular}{|l|}
$437-537$ \\
Kustria \\
Beigica \\
Est. Unidos \\
Franca \\
Irl. do Norte \\
Itaília \\
Hova Zeiândia
\end{tabular} & $\begin{array}{l}537 \curvearrowleft 637 \\
\text { Alenanha Fed. } \\
\text { Canada } \\
\text { Escócia } \\
\text { Finlāindia } \\
\text { Japso }\end{array}$ & $\begin{array}{l}637 \vdash 737 \\
\text { Aus trallia } \\
\text { Holanda } \\
\text { Ingl. E Gales } \\
\text { Isiândia les } \\
\text { Luxenburgo } \\
\text { Noruega } \\
\text { Suica }\end{array}$ & $\begin{array}{l}737 \vdash \\
\text { Dinamarca } \\
\text { Suécia }\end{array}$ \\
\hline $\begin{array}{l}z=2,7583 x_{1}+3,4044 x_{2}+ \\
+x_{4}-3,3799 x_{6}\end{array}$ & -117 & $\begin{array}{l}117-209 \\
\text { Chile } \\
\text { Egito } \\
\text { Guatema la } \\
\text { Gutana } \\
\text { Ihas Mavicicias } \\
\text { Mexico } \\
\text { Trinidade Tobago } \\
\text { Uruguai }\end{array}$ & $\begin{array}{l}209 \curvearrowleft 302 \\
\text { Barbados } \\
\text { Costa Rica } \\
\text { Israel } \\
\text { Iugosiávia } \\
\text { Panamáa } \\
\text { Polónia } \\
\text { Portugal } \\
\text { Romênia }\end{array}$ & $\begin{array}{l}302-394 \\
\text { Bulgāria } \\
\text { Espanha } \\
\text { Grectia } \\
\text { Hong Kong } \\
\text { Hungria } \\
\text { Irlanda } \\
\text { Singapura }\end{array}$ & $\begin{array}{l}394-487 \\
\text { Austria } \\
\text { Bêlgica } \\
\text { Est. Unidos } \\
\text { Franca } \\
\text { Irl. do Norte } \\
\text { Itâli ia. } \\
\text { Wova Zeländia }\end{array}$ & 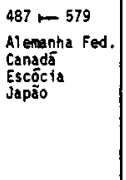 & 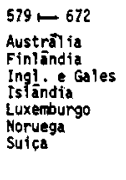 & $\begin{array}{l}672 \text { म } \\
\text { Dinamarca } \\
\text { Holanda } \\
\text { Suécia }\end{array}$ \\
\hline $\begin{array}{l}z=2,5261 x_{1}+3,6214 x_{2}+ \\
+x_{4}+0,5236 x_{5}= \\
-3,5779 x_{6}\end{array}$ & -162 & $\begin{array}{l}162-255 \\
\text { Chile } \\
\text { Egito } \\
\text { Guatenala } \\
\text { Guiana } \\
\text { Ihas Maurícias } \\
\text { Wexico } \\
\text { Trinidad e Tobago } \\
\text { Uruguat } i\end{array}$ & $\begin{array}{l}255,349 \\
\text { Barbados } \\
\text { Costa Rica } \\
\text { Irlanda } \\
\text { Israel } \\
\text { Iugos läria } \\
\text { Panama } \\
\text { Polónia } \\
\text { Portugal } \\
\text { Romenia }\end{array}$ & $\begin{array}{l}349-442 \\
\text { Bulgariaa } \\
\text { Espanha } \\
\text { Grecia } \\
\text { Hong Kong } \\
\text { Hungria } \\
\text { trlanda do Norte } \\
\text { Singapura }\end{array}$ & $\begin{array}{l}442 \text { - } 536 \\
\text { Austria } \\
\text { BẼgica } \\
\text { Est. Unidos } \\
\text { Franca } \\
\text { Itâlis } \\
\text { Hova Zelandia }\end{array}$ & $\begin{array}{l}536 \_629 \\
\text { Al manha Fed. } \\
\text { Canadá } \\
\text { Escócia } \\
\text { Finlandia }\end{array}$ & $\begin{array}{l}629 \text { - } 723 \\
\text { Austräl ia } \\
\text { Ingl. o Gales } \\
\text { Islândia } \\
\text { Japao } \\
\text { Luxemburgo } \\
\text { Noruega } \\
\text { Suica }\end{array}$ & $\begin{array}{l}723 \curvearrowleft \\
\text { Dinamarca } \\
\text { Holanda } \\
\text { Suécia }\end{array}$ \\
\hline $\begin{array}{l}z=2,5436 x_{1}+3,6108 x_{2}- \\
\quad-0,6272 x_{3}+x_{4}+ \\
+0,5931 x_{5}=3,4253 x_{6}\end{array}$ & -129 & $\begin{array}{l}\text { 129-223 } \\
\text { Chile } \\
\text { Egito } \\
\text { Guatema la } \\
\text { Guiana } \\
\text { 1has Mariclas } \\
\text { Mexico } \\
\text { Trinidad e Tobago } \\
\text { Uruguai }\end{array}$ & $\begin{array}{l}\text { 223 - } 317 \\
\text { Barbados } \\
\text { Costa Rica } \\
\text { Irlanda } \\
\text { israel } \\
\text { Iugoslaviala } \\
\text { Panama } \\
\text { Poloniala } \\
\text { Portugal } \\
\text { Romenia }\end{array}$ & 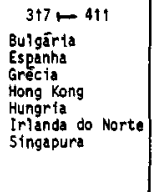 & $\begin{array}{l}411-505 \\
\text { Austria } \\
\text { Bêlgica } \\
\text { Est. Unidos } \\
\text { Franca } \\
\text { Itâtia } \\
\text { Nova Zeländia }\end{array}$ & $\begin{array}{l}505 \vdash 599 \\
\text { Alemanha Fed. } \\
\text { Canadé } \\
\text { Escócida } \\
\text { Fintändia }\end{array}$ & 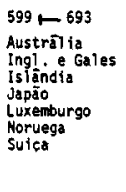 & $\begin{array}{l}693 \curvearrowleft \\
\text { Dinamarca } \\
\text { Holanda } \\
\text { Suécia }\end{array}$ \\
\hline
\end{tabular}

Para comparação da ordenação, baseada na equação escolhida, com as resultantes das demais equações, foi elaborada a Tabela 8 . Como se pode verificar, a ordenação baseada na equação que inclui a recíproca de apenas um indicador, o CMI, discrepa substancialmente das demais, evidenciando, como já foi referido, a falta de paralelismo no comportamento dos indicadores estudados.
Mesmo com as recíprocas de dois indicadores incluídos na equação, a ordenação ainda difere apreciavelmente das demais.

A partir da equação com três recíprocas, ou seja, a que foi considerada mais adequada, as diferenças observadas com as demais são em pequeno número e limitadas a variações de uma classe. Deve-se notar que as mudanças da classe $H$ 
NOVO, N.F. et al. Ordenação de populações em amplas classes de nível de saúde, segundo um indicador abrangente definido por uma função discriminante linear. Rev. Saúde públ., S. Paulo, 19:344-63, 1985.

\section{TABELA 8}

Classificação dos países. segundo as equações discriminantes de que resultam os valores de $Z$.

\begin{tabular}{|c|c|c|c|c|c|c|}
\hline PAIS & $z=9,2664 x_{1}$ & $z=2,9140 x_{1}+x_{4}$ & $\begin{array}{l}z=2,8950 x_{1}+ \\
+2,0599 x_{2}+x_{4}\end{array}$ & $\begin{array}{l}z=2,7583 x_{1}+3,4044 x_{2}+ \\
+x_{4}-3,3799 x_{6}\end{array}$ & $\begin{array}{c}z=2,5261 x_{1}+3,6214 x_{2}+x_{4}+ \\
+0,5236 x_{5}=3,5779 x_{6}\end{array}$ & $\begin{array}{l}2=2,5436 x_{1}+3,6108 x_{2} \\
-0,6272 x_{3}+x_{4}+ \\
+0,5931 x_{5}+3,4253 x_{6}\end{array}$ \\
\hline Alemanha Federal & D & D & c & c & c & c \\
\hline Austrāl ia & $c$ & B & B & B & B & B \\
\hline Austria & D & $\mathrm{D}$ & $D$ & D & D & $D$ \\
\hline Barbados & $\mathbf{F}$ & $F$ & $\mathbf{F}$ & $F$ & $\mathbf{F}$ & $\mathbf{F}$ \\
\hline BËlgica & D & D & $D$ & D & D & D \\
\hline Bulgāría & $\mathrm{F}$ & $E$ & $E$ & $E$ & $\mathbf{E}$ & $E$ \\
\hline Canadã & D & C & $c$ & c & C & c \\
\hline chile & G & G & 6 & 6 & $G$ & G \\
\hline Costa Rica & $\mathbf{F}$ & $F$ & $\mathbf{F}$ & $F$ & $\mathbf{F}$ & $\mathrm{F}$ \\
\hline Dinamarca & B & A & A & A & A & A \\
\hline Egito & $H$ & $G$ & H & G & G & 6 \\
\hline Escōcía & C & $c$ & c & c & c & C \\
\hline Espanha & $\mathrm{E}$ & E & $\mathrm{E}$ & $E$ & $\mathrm{E}$ & $\mathrm{E}$ \\
\hline Estados Unidos & D & D & D & $D$ & D & $D$ \\
\hline Finländia & A & B & $c$ & B & c & c \\
\hline França & c & D & D & D & D & D \\
\hline Grēcia & $\mathbf{E}$ & $\mathbf{E}$ & $\mathrm{E}$ & $\mathbf{E}$ & $E$ & $E$ \\
\hline Guatema la & H & H & $H$ & G & G & G \\
\hline Guiana & G & G & G & G & $G$ & G \\
\hline Hoianda & B & A & B & A & A & A \\
\hline Hong Kong & D & $E$ & E & $E$ & $E$ & $E$ \\
\hline Hungría & $\mathbf{F}$ & $F$ & E & E & E & $\mathbf{E}$ \\
\hline Ilhas Mouricias & G & G & 6 & G & G & $G$ \\
\hline Inglaterra e Gales & D & B & B & B & B & B \\
\hline Irlanda & $E$ & E & $E$ & E & $\mathbf{F}$ & $\mathbf{F}$ \\
\hline Irlanda do Norte & E & D & $D$ & D & E & $\mathbf{E}$ \\
\hline Islândia & A & A & B & B & B & B \\
\hline Israel & $E$ & E & $E$ & $F$ & $F$ & $F$ \\
\hline I & $E$ & D & D & $D$ & D & D \\
\hline Iugosiēvia & G & G & $\mathbf{F}$ & $F$ & $\mathbf{F}$ & $\mathbf{F}$ \\
\hline Japāo & A & $c$ & $c$ & $c$ & B & B \\
\hline Luxemburgo & B & B & B & B & B & B \\
\hline Mêxico & G & G & H & 6 & G & 6 \\
\hline Noruega & B & B & B & B & B & B \\
\hline Mova Zelāndia & D & $c$ & D & $D$ & D & D \\
\hline Panamá & $\mathbf{F}$ & $F$ & $G$ & $\mathbf{F}$ & $\mathbf{F}$ & $\mathbf{F}$ \\
\hline Polônía & $\mathbf{F}$ & $\mathrm{F}$ & $\mathbf{F}$ & $\mathbf{F}$ & $\mathbf{F}$ & $F$ \\
\hline Portugal & $\mathrm{F}$ & $\mathrm{F}$ & $\mathrm{F}$ & $\mathrm{F}$ & $\mathbf{F}$ & $\mathbf{F}$ \\
\hline Romên ia & $\mathrm{F}$ & $F$ & $F$ & $F$ & $\mathrm{~F}$ & $\mathrm{~F}$ \\
\hline Singapura & D & $E$ & E & $E$ & E & $\mathbf{E}$ \\
\hline Suêcia & A & A & A & A & A & A \\
\hline Suiça & B & B & B & B & B & B \\
\hline Trinidad • Tobago & $\mathrm{F}$ & G & G & G & $G$ & G \\
\hline Urugua $i$ & 6 & $G$ & 6 & G & $G$ & G \\
\hline
\end{tabular}

para a classe $G$ traduzem a incapacidade das equações, com quatro ou mais variáveis, de produzirem valores classificáveis como $\mathrm{H}$.

$$
X_{2}=\frac{1000}{C N} e
$$

Lembrando que:

$$
\mathrm{X}_{1}=\frac{1000}{\mathrm{CMI}} \quad \mathrm{X}_{4}=\frac{1000}{\text { CMDTp }}
$$


NOVO, N.F. et al. Ordenação de populações em amplas classes de nível de saúde, segundo um indicador abrangente definido por uma função discriminante linear. Rev. Saúde públ., S. Paulo, 19:344-63, 1985.

a equação:

$$
Z=2,8950 X_{1}+2,0599 X_{2}+X_{4}
$$
pode ser reformulada, para fins operacionais, visando a tornar desnecessário o cálculo das recíprocas, assumindo, com grau suficiente de aproximação, a forma:

$$
\mathrm{Z}=\frac{2895}{\mathrm{CMI}}+\frac{2060}{\mathrm{CN}}+\frac{1000}{\mathrm{CMDTp}}
$$

\section{CONCLUSĀO}

Considerando os elementos colhidos no estudo dos dados apresentados e a discussão dos resultados obtidos, sendo:
$\mathrm{CMI}=$ coeficiente de mortalidade infantil;
$\mathrm{CN} \quad=$ coeficiente de natalidade; $\mathrm{e}$
CMDTp $=$ coeficiente de mortalidade por doenças transmissíveis padronizado pela população mediana definida;

o valor $Z$, na equação da função discriminante linear,

$$
\mathrm{Z}=\frac{2895}{\mathrm{CMI}}+\frac{2060}{\mathrm{CN}}+\frac{1000}{\text { CMDTp }}
$$

pode ser utilizado como indicador abrangente que permite a ordenação de populações em amplas classes de nível de saúde.

NOVO, N.F, et al. [Ranking of populations in broad classes of health levels according to a comprehensive indicador defined by a linear discriminant function]. Rev. Saude públ., S. Paulo, $19: 344-63,1985$.

ABSTRACT: There are, very often, considerable discrepancies when countries are ranked according to the values of each of the common health indicators. By the use of computed linear discriminant functions the authors developed a single indicator designed to convey the information gathered from the following health indicators: lif a expectancy at birth (LE), birth rate (BR), infant mortality rate (IMR), quantified indicator of Guedes (GI), general mortality rate (GMR) and mortality rate (MR) by infective and parasitic diseases (MRIPD), the last two age adjusted. For the construction of this adjustment a median standard population was suggested and used, each age group contributed with the average of the percentages of participation of the group in the composition of the population of each one of the 44 countries studied. These were those for which it was possible to get reliable data for the years around 1980 . The contrasted groups in computing discriminant functions, each one consisting of 12 countries, were defined according to a criterion based on the rank of the sum of the normal reduced deviations calculated for the distributions of the values for each indicator. For the computation of discriminant function equations by the stepwise technique, reciprocal transformation was used for the four indicators expressed as ratios and for the other two their face values were used. Critical analysis of results as shown that the formula: $Z=\frac{2895}{\mathrm{IMR}}+\frac{2060}{\mathrm{BR}}+\frac{1000}{\text { MRIPD }}$, can be used as a comprehensve indicator allowing the ranking of countries in broad classes of health levels, as follows: A-737 or more; Denmark and Sweden; B - 637 $\vdash$ 737: Australia, Netherland, England and Wales, Iceland, Luxembourg, Norway and Switzerland; C - $537 \vdash 637$ : Federal Republic of Germany, Canada, Scotland, Finland and Japan; D-437 - 537: Austria, Belgium, United States, France, Northern Ireland, Italy and New Zealand; E - $337 \vdash$ 437: Bulgaria, Spain, Greece, Hong Kong, Hungary, Ireland, Israel and Singapore; F - $237 \vdash 337$ : Barbados, Costa Rica, Yugoslavia, Poland, Portugal and Romania; $G-137 \vdash 237$ : Chile, Guyana, Mauritius, Panama, Trinidad and Tobago and Uruguay; $\mathrm{H} \cdot<137$ : Egygt, Guatemala and Mexico.

UNITERMS: Health status indicators. Comprehensive health indicator. Linear discriminant function. Median standard population. 
NOVO, N.F. et al. Ordenação de populações em amplas classes de nível de saúde, segundo um indicador abrangente definido por uma função discriminante linear. Rev. Saúde pübl. S. Paulo, 19:344-63, 1985.

\section{REFERÊNCIAS BIBLIOGRÁFICAS}

1. ARMITAGE, P. Statistical methods in medical research. Oxford, Blackwell, 1971.

2. DEMOGRAPHIC YEARBOOK. (United Nations). New York, v. 30, 1978; v. 31, 1979; v. 32, 1980.

3. GOULDEN, C.H. The discriminant function. In: Goulden, C.H. Methods of statistical analysis. 2nd ed. New York, Wiley, 1952. p. 378-93.

4. GUEDES, J.S. Contribuição para o estudo da evolução do nível de saúde do Estado de São Paulo: análise das regiões administrativas (1950-1970). São Paulo, 1972. [Tese de Doutoramento Faculdade de Saúde Pública da USP]

5. KELLEY, T.L. The selection of upper and lower groups for the validation of test items. J. educ. Psychol, 30:17-24, 1939.

6. MORAES, N.L. Níveis de saúde de coletividades brasileiras. Rev. Serv. publ., Rio de Janeiro, 10:403-97, 1959.
7. SHRYOCK, H.S. \& SIEGEL, J.S. The methods and materials of demography. Washington, D.C., U.S. Department of Commerce, 1973 , v. 2.

8. SNEDECOR, G.W. \& COCHRAN, W.G. Metodos estadisticos. $6 .^{\mathrm{a}}$ ed. Mexico, Continental, 1971.

9. SOKAL, R.R. \& ROHLF, F.J. Biometry: the principles and practice of statistics in biological research. San Francisco, Freeman, 1969.

10. SWAROOP, S. \& UEMURA, K. Proportional mortality of 50 years and above: a suggested indicator of the component "health, including demographic conditions" in the measurement of levels of living. Bull. Wld Hlth Org., 17:439-81, 1957.

11. WORLD HEALTH STATISTICS ANNUAL. (World Health Organization). Geneva, 1977; 1978; 1979; 1980; 1981; 1982.

Recebido para publicação em 22/02/1985

A provado para publicação em 21/05/1985 